Elsevier Editorial System(tm) for Journal of Computational and Applied Mathematics Manuscript Draft

Manuscript Number: CAM-D-17-01746R1

Title: \$r\$-norm bounds and metric properties for zero loci of real analytic functions

Article Type: Research Paper

Section/Category: 68w30 (Symbolic computation and algebraic computation)

Keywords: Matrix norms equivalence; zero locus of real analytic

functions, crossing area conditions, \$r\$-norm distance

Corresponding Author: Professor Juan Rafael Sendra, Prof.

Corresponding Author's Institution: Universidad de Alcala

First Author: Juan Rafael Rafael Sendra, Prof.

Order of Authors: Juan Rafael Rafael Sendra, Prof.; Mauro Beltrametti; Laura Torrente

Abstract: We consider the problem of deciding whether or not a zero locus, \$X\$, of multivariate real analytic functions

crosses a given \$r\$-norm ball in the real \$n\$-dimensional affine space. We perform a local study of the problem, and we provide both necessary and sufficient conditions to answer the question. Our conditions derive from the analysis of differential geometric properties of $\$ x \$$ at the center of the ball. An algorithm to evaluate \$r\$-norms distances is proposed. 


\title{
$r$-norm bounds and metric properties for zero loci of real analytic functions*
}

\author{
M. Torrente, M.C. Beltrametti, and J.R. Sendra
}

\begin{abstract}
We consider the problem of deciding whether or not a zero locus, $X$, of multivariate real analytic functions crosses a given $r$-norm ball in the real $n$-dimensional affine space. We perform a local study of the problem, and we provide both necessary and sufficient conditions to answer the question. Our conditions derive from the analysis of differential geometric properties of $X$ at the center of the ball. An algorithm to evaluate $r$-norms distances is proposed.
\end{abstract}

\section{Introduction}

Let us say that $X$ is a subset of $\mathbb{R}^{n}$ and $p \in \mathbb{R}^{n} \backslash X$, where $\mathbb{R}^{n}$ is endowed with a metric $\|\cdot\|_{r}$. We are interested in finding conditions on a positive number $\varepsilon$ to ensure that the closed $r$-ball (or a subset of it) centered at $p$ and of radius $\varepsilon$ either intersects $X$ or does not intersect $X$. Indeed, this turns out to be useful in practical applications, for instance, in the setting of the Hough transform, a standard technique to detect curves in images (e.g., see [2], 16], [17], [18]), or for evaluating the performance of robots that are required to follow a desired reference path (see [13]), or even in the field of robot motion planning (see [3]).

In the first case, in the application of the Hough transform method two main steps have to be considered: the discretization in cells of a parameter space, and a voting process to choose the most suitable cell. In this situation, $X$ is a hypersurface in the parameter space, that is, the Hough transform of a point of the image space, and $p$ represents the center of a given cell, defined in $\infty$-norm. In the second case, $p$ is the vehicle position which is taken as the center of a 2-ball, and $X$ represents the reference path. Finally, in the third case, the point $p$ describes the position of the robot and $X$ the region of interest in the workspace.

In order to approach the problem, we take $X$ as the zero locus of a finite collection of real functions. In [13] and [16] the problem is solved for the case where $X$ is an algebraic hypersurface and the balls are taken in 2 - and $\infty$ - norms, respectively. In this paper, inspired by the papers quoted above, we deal with a more general situation, namely, $X$ is the zero locus of a finite collection of real analytic functions and the balls are taken in $r$-norms, with $r \in[1, \infty]$.

\footnotetext{
*2010 Mathematics Subject Classification. Primary 30C15; 15A60; 68W30; Secondary 14Q99

Keywords and phrases. Matrix norms equivalence; zero locus of real analytic functions, crossing area conditions, $r$-norm distance
} 
To be more precise, consider multivariate real functions $f_{j}=f_{j}(\boldsymbol{x}), j=1, \ldots, m$, $m \leq n$, being analytic on an open convex set $U \subseteq \mathbb{R}^{n}$, where $\boldsymbol{x}=\left(x_{1}, \ldots, x_{n}\right)$ denotes cartesian coordinates on $\mathbb{R}^{n}$. Let $X \subset \cap_{j=1}^{m} \operatorname{dom}\left(f_{j}\right)$ be the zero locus of the $f_{j}$ 's. Let $r \in[1, \infty]$. The $(r, \varepsilon)$-ball $\mathbf{B}_{r}(p, \varepsilon)$ centered at a point $p \in U$ is defined as

$$
\mathbf{B}_{r}(p, \varepsilon)=\left\{\boldsymbol{x} \in \mathbb{R}^{n} \text { such that }\left\|(\boldsymbol{x}-p)^{t}\right\|_{r} \leq \varepsilon\right\} .
$$

We choose the threshold $\varepsilon$ such that $\mathbf{B}_{r}(p, \varepsilon) \subset U$, and we consider any subset $\mathbf{C}_{r}(p, \varepsilon)$ of $\mathbf{B}_{r}(p, \varepsilon)$ such that the interior of both is the same. We refer to $\mathbf{C}_{r}(p, \varepsilon)$ as a $(r, \varepsilon)$ cell centered at $p$ (see Definition 3.1). In Section 3, we provide necessary numerical conditions for $X$ to cross $\mathbf{C}_{r}(p, \varepsilon)$ (see Theorem 3.2). While in Section 4 we provide sufficient conditions (see Theorem 4.6).

Unfortunately, the above conditions do not fit together to give "if and only if" statements. However, because of the local nature of the results, a more accurate analysis, performed by iteratively considering a subdivision of the $(\infty, \varepsilon)$-ball $\mathbf{B}_{\infty}(p, \varepsilon)$ centered at $p$, which clearly contains the given $(r, \varepsilon)$-cell $\mathbf{C}_{r}(p, \varepsilon)$, may overcome the problem. Section 5 is devoted to a detailed discussion of that issue, and several examples show the effectiveness of the method, summed up in the Iterative Crossing Cell Algorithm.

In Section 1 we recall some background material, while in Section 2 we survey properties of matrix $r$-norms we need.

\section{Background material}

In this section we collect some definitions and basic facts about multivariable differential calculus used throughout the paper. For more details we refer to [1, Chapter 12] and [4, Chapter 4]. Let $U \subseteq \mathbb{R}^{n}$ be an open convex set, and let $x_{1}, \ldots, x_{n}$ be coordinates in $\mathbb{R}^{n}$. Most of the times we use for simplicity the notation $\boldsymbol{x}=\left(x_{1}, \ldots, x_{n}\right)$. Let

$$
\mathcal{A}(U)=\left\{f: U \subseteq \mathbb{R}^{n} \rightarrow \mathbb{R} \mid f \text { is analytic in } U\right\}
$$

be the set of all real functions $f(\boldsymbol{x})$ being analytic in $U$. We recall that functions in $\mathcal{A}(U)$ are infinitely differentiable, and their derivatives are again analytic in $U$. This will, in particular, ensure that the entries of the Hessian of $f \in \mathcal{A}(U)$ are continuous functions in $U$, and hence the quantity $\mathrm{H}^{r}$, as in (7) in Section 3, will be well-defined. In addition, we also recall that $\mathcal{A}(U)$ is an integral domain. This property will be used, for instance, when working with the Moore-Penrose inverse of matrices over $\mathcal{A}(U)$. Let $P$ denote the multivariate polynomial ring $\mathbb{R}[\boldsymbol{x}]=\mathbb{R}\left[x_{1}, \ldots, x_{n}\right]$, with $x_{1}, \ldots, x_{n}$ indeterminates. Note that $P \subset \mathcal{A}(U)$.

Given $\boldsymbol{\alpha}=\left(\alpha_{1}, \ldots, \alpha_{n}\right) \in \mathbb{N}^{n}$, we denote by $|\boldsymbol{\alpha}|$ the number $\alpha_{1}+\cdots+\alpha_{n}$, by $\boldsymbol{\alpha}$ ! the number $\alpha_{1} ! \ldots \alpha_{n} !$, and by

$$
\frac{\partial^{\alpha} f}{\partial \boldsymbol{x}^{\alpha}}:=\frac{\partial^{|\boldsymbol{\alpha}|} f}{\partial x_{1}^{\alpha_{1}} \ldots \partial x_{n}^{\alpha_{n}}}
$$

the $\boldsymbol{\alpha}$-partial derivative of $f \in \mathcal{A}(U)$. Moreover, following the standard notation, we denote by

$$
\operatorname{Jac}_{f}(\boldsymbol{x}):=\left(\frac{\partial f}{\partial x_{1}}, \ldots, \frac{\partial f}{\partial x_{n}}\right)
$$


the Jacobian (or gradient) of $f$, and by

$$
H_{f}(\boldsymbol{x}):=\left(\frac{\partial^{2} f}{\partial x_{i} \partial x_{j}}\right)_{i, j=1, \ldots, n}
$$

the $n \times n$ Hessian matrix of $f \in \mathcal{A}(U)$. More generally, for a vector valued real function $F=\left(f_{1}, \ldots, f_{m}\right)$, where $f_{i} \in \mathcal{A}(U)$, we denote by

$$
\operatorname{Jac}_{F}(\boldsymbol{x})=\left(\begin{array}{ccc}
\frac{\partial f_{1}}{\partial x_{1}} & \cdots & \frac{\partial f_{1}}{\partial x_{n}} \\
\vdots & & \vdots \\
\frac{\partial f_{m}}{\partial x_{1}} & \cdots & \frac{\partial f_{m}}{\partial x_{n}}
\end{array}\right)
$$

the $m \times n$ Jacobian matrix of $F$, and by

$$
H_{F}(\boldsymbol{x}):=\left(\begin{array}{c}
H_{f_{1}}(\boldsymbol{x}) \\
\vdots \\
H_{f_{m}}(\boldsymbol{x})
\end{array}\right)
$$

the $m n \times n$ Hessian matrix of $F$.

By abuse of notation, if $M$ is a $m \times 1$ matrix whose entries are real functions in $\boldsymbol{x}$, we denote by $\operatorname{Jac}_{M}(\boldsymbol{x})$ the Jacobian of $M^{t}$ and by $H_{M}(\boldsymbol{x})$ the Hessian of $M^{t}$.

Proposition 1.1 (Taylor's theorem) Let $U \subseteq \mathbb{R}^{n}$ be an open convex set and let $f=$ $f(\boldsymbol{x}): U \rightarrow \mathbb{R}$ be a real $(k+1)$-th differentiable function. Let $p$ be a point of $U$. Then, for each $\boldsymbol{x} \in U$, one has $f(\boldsymbol{x})=p_{k}(\boldsymbol{x})+R_{k}(\boldsymbol{x})$, where

$$
p_{k}(\boldsymbol{x})=\sum_{|\boldsymbol{\alpha}| \leq k} \frac{1}{\boldsymbol{\alpha} !} \frac{\partial^{\boldsymbol{\alpha}} f}{\partial \boldsymbol{x}^{\boldsymbol{\alpha}}}(p)(\boldsymbol{x}-p)^{\boldsymbol{\alpha}} \quad \text { and } \quad R_{k}(\boldsymbol{x})=\sum_{|\boldsymbol{\alpha}|=k+1} \frac{1}{\boldsymbol{\alpha} !} \frac{\partial^{\boldsymbol{\alpha}} f}{\partial \boldsymbol{x}^{\boldsymbol{\alpha}}}(\xi)(\boldsymbol{x}-p)^{\boldsymbol{\alpha}}
$$

for some point $\xi \in \mathbb{R}^{n}$ of the line segment from $p$ to $\boldsymbol{x}$.

\section{A survey on matrix $r$-norms}

In this section we recall basic definitions and concepts from numerical algebra used throughout the paper, very often without explicitly refer to them. We start recalling the definition of different norms on the space of matrices and their basic properties (for proofs and more details we also refer to [8] and [19]).

For $m, n$ positive integers, we let $\operatorname{Mat}_{m \times n}(\mathbb{R})$ be the set of $m \times n$ matrices with entries in $\mathbb{R}$; if $m=n$ we simply write $\operatorname{Mat}_{n}(\mathbb{R})$. For any $M \in \operatorname{Mat}_{m \times n}(\mathbb{R})$, we will denote by $M^{t}$ its transpose.

Definition 2.1 Let $v$ be an element of $\operatorname{Mat}_{n \times 1}(\mathbb{R})$ and let $r \geq 1$ be a real number. Set $v^{t}:=\left(v_{1}, \ldots, v_{n}\right)$. The $r$-norm ${ }^{1}$ of $v$ is defined by

$$
\|v\|_{r}:=\left(\sum_{i=1}^{n}\left|v_{i}\right|^{r}\right)^{\frac{1}{r}}
$$

If $r \rightarrow \infty$, the $r$-norm approaches the $\infty$-norm defined by $\|v\|_{\infty}:=\max _{i=1, \ldots, n}\left\{\left|v_{i}\right|\right\}$.

\footnotetext{
${ }^{1}$ We will only use matrix norms; however, let us mention that in the literature one also refers to this norm as the " $r$-norm of the vector $\left(v_{1}, \ldots, v_{n}\right)$ in $\mathbb{R}^{n}$ ".
} 
We need the following facts about $r$-norms: Hölder's inequality and an equivalence result.

Proposition 2.2 (Hölder's inequality) Let either $q>r \geq 1$ be real numbers such that $\frac{1}{q}+\frac{1}{r}=1$ or $q=\infty$ and $r=1$. Then for each $v, w \in \operatorname{Mat}_{n \times 1}(\mathbb{R})$ we have the inequality

$$
\left|v^{t} w\right| \leq\|v\|_{q}\|w\|_{r}
$$

In the sequel, we use the following notation. Consider the real interval $[1, \infty)$ and set $[1, \infty]=[1, \infty) \cup\{\infty\}$.

Proposition $2.3\left[6\right.$, Chapter 8] Let $r, s \in[1, \infty]$ with $r \neq s$. For each $v \in \operatorname{Mat}_{n \times 1}(\mathbb{R})$ the following inequalities hold:

1. $\|v\|_{s} \leq\|v\|_{r} \leq n^{\frac{1}{r}-\frac{1}{s}}\|v\|_{s}$ if $1 \leq r<s \leq \infty$.

2. $n^{\frac{1}{r}-\frac{1}{s}}\|v\|_{s} \leq\|v\|_{r} \leq\|v\|_{s}$ if $1 \leq s<r \leq \infty$.

A given $r$-norm on $\operatorname{Mat}_{n \times 1}(\mathbb{R})$ induces a norm on $\operatorname{Mat}_{m \times n}(\mathbb{R}), r \in[1, \infty]$ (see $[8$, formula (2.3.2)]). We recall the definition and some basic properties.

Definition 2.4 Let $M=\left(m_{i j}\right)$ be a matrix in $\operatorname{Mat}_{m \times n}(\mathbb{R})$. The r-matrix norm is the norm on $\operatorname{Mat}_{m \times n}(\mathbb{R})$ defined by the formula

$$
\|M\|_{r}:=\max _{\|v\|_{r}=1}\|M v\|_{r}
$$

where $v \in \operatorname{Mat}_{n \times 1}(\mathbb{R})$.

In particular, one has $\|M\|_{1}=\max _{j=1, \ldots, n}\left\{\sum_{i=1, \ldots, m}\left|m_{i j}\right|\right\}$, and $\|M\|_{2}=\sqrt{\lambda\left(M^{t} M\right)}$ where $\lambda\left(M^{t} M\right)$ denotes the biggest eigenvalue of $M^{t} M$. While, if $r \rightarrow \infty$, the matrix $r$ norm approaches the $\infty$-matrix norm defined by $\|M\|_{\infty}:=\max _{i=1, \ldots, m}\left\{\sum_{j=1, \ldots, n}\left|m_{i j}\right|\right\}$. However, as pointed out in the literature (for instance, see [7]), no explicit formula is available for $\|\cdot\|_{r}$ (unless $r=1,2, \infty$ as mentioned above), but only certified estimates.

Remark 2.5 Let us point out the following consequence of Definition 2.4. For any matrix $M \in \operatorname{Mat}_{m \times n}(\mathbb{R})$, one has $\|M\|_{1}=\left\|M^{t}\right\|_{\infty}$.

Next, let us introduce one more matrix norm we need.

Definition 2.6 Let $M=\left(m_{i j}\right)$ be a matrix in $\operatorname{Mat}_{m \times n}(\mathbb{R})$. The max-norm is the norm on $\operatorname{Mat}_{m \times n}(\mathbb{R})$ defined by

$$
\|M\|_{\max }:=\max _{i, j}\left|m_{i j}\right|
$$

Note that, via the natural identification of $\operatorname{Mat}_{m \times n}(\mathbb{R})$ with $\operatorname{Mat}_{m n \times 1}(\mathbb{R})$, a matrix $M \in \operatorname{Mat}_{m \times n}(\mathbb{R})$ can be viewed as an element, that we will denoted by $M^{(\mathbf{v})}$ to avoid confusion, of $\operatorname{Mat}_{m n \times 1}(\mathbb{R})$. Moreover,

$$
\|M\|_{\max }=\left\|M^{(\mathbf{v})}\right\|_{\infty} .
$$


We recall that the norms introduced in Definition 2.4 are equivalent norms. We use the following notation. Given $r, s \in[1, \infty]$, we denote by $\alpha_{r s}^{m \times n}$ and $\beta_{r s}^{m \times n}$ as

$$
\begin{aligned}
& \beta_{r s}^{m \times n}=\inf \left\{\beta \in \mathbb{R} \mid\|M\|_{r} \leq \beta\|M\|_{s} \forall M \in \operatorname{Mat}_{m \times n}(\mathbb{R})\right\}, \\
& \alpha_{r s}^{m \times n}=\sup \left\{\alpha \in \mathbb{R} \mid \alpha\|M\|_{s} \leq\|M\|_{r} \forall M \in \operatorname{Mat}_{m \times n}(\mathbb{R})\right\} .
\end{aligned}
$$

Proposition 2.7 Let $r, s \in[1, \infty]$, and consider the matrix norms $\|\cdot\|_{r}$ and $\|\cdot\|_{s}$ on the space $\operatorname{Mat}_{m \times n}(\mathbb{R})$ of matrices. Then,

$$
\beta_{r s}^{m \times n}= \begin{cases}m^{\frac{1}{r}-\frac{1}{s}} & \text { if } r \leq s, \\ n^{\frac{1}{s}-\frac{1}{r}} & \text { if } r \geq s .\end{cases}
$$

Proof. Obviously, $\beta_{r s}^{m \times n}=1$ if $r=s$. Let $r<s \leq \infty$ and let $M \in \operatorname{Mat}_{m \times n}(\mathbb{R})$. From [7, formula (1.6)] (see also [12]) we know that

$$
\max _{M \neq 0} \frac{\|M\|_{r}}{\|M\|_{s}}=\left(\max _{\mathbf{0} \neq v \in \operatorname{Mat}_{m \times 1}(\mathbb{R})} \frac{\|v\|_{r}}{\|v\|_{s}}\right)\left(\max _{\mathbf{0} \neq v \in \operatorname{Mat}_{n \times 1}(\mathbb{R})} \frac{\|v\|_{s}}{\|v\|_{r}}\right) .
$$

Note that $\max _{\mathbf{0} \neq v \in \operatorname{Mat}_{m \times 1}(\mathbb{R})} \frac{\|v\|_{r}}{\|v\|_{s}}=m^{\frac{1}{r}-\frac{1}{s}}$. Indeed, from the second inequality of Proposition $2.3(1)$, it follows that $\max _{\mathbf{0} \neq v \in \operatorname{Mat}_{m \times 1}(\mathbb{R})} \frac{\|v\|_{r}}{\|v\|_{s}} \leq m^{\frac{1}{r}-\frac{1}{s}}$, with equality for $v=(1, \ldots, 1)^{t}$, whence the claimed assertion. Similarly, from the first inequality of Proposition 2.3 (1) it follows that $\max _{\mathbf{0} \neq v \in \mathrm{Mat}_{n \times 1}(\mathbb{R})}\|v\|_{s} \leq 1$, with equality for $v=(1,0, \ldots, 0)^{t}$, so that $\max _{\mathbf{0} \neq v \in \operatorname{Mat}_{n \times 1}(\mathbb{R})}\|v\|_{s}=1$. Therefore

$$
\max _{M \neq \mathbf{0}} \frac{\|M\|_{r}}{\|M\|_{s}}=m^{\frac{1}{r}-\frac{1}{s}}
$$

Thus, by combining (3) and (4), we conclude that the best possible value for $\beta_{r s}^{m \times n}$ is $m^{\frac{1}{r}-\frac{1}{s}}$. The same argument gives the desired result in the case $\infty \geq r>s$. $\quad$ Q.E.D.

The following is an immediate consequence of Proposition 2.7.

Corollary 2.8 Let $r, s \in[1, \infty]$ and $m, n \in \mathbb{N} \backslash\{0\}$. Then:

1. $\beta_{r s}^{m \times n} \geq 1$.

2. $\beta_{r s}^{m \times n} \geq \beta_{r s}^{m \times 1}$.

Proof. Statement 1) clearly follows from Proposition 2.7. To prove assertion 2) we observe that, by Proposition 2.7, if $r \leq s$ then $\beta_{r s}^{m \times n}=\beta_{r s}^{m \times 1}$, and if $r \geq$ then $\beta_{r s}^{m \times 1}=1$. Thus the result follows from Statement 1).

Q.E.D.

In the next two propositions, we specify the equivalence of the matrix norms $\|\cdot\|_{\max }$ and $\|\cdot\|_{2}$, and we recall the sub-multiplicative property (see [8, $\left.\S 2.3 .1\right]$ ) for $r$-norms.

Proposition 2.9 For each $M \in \operatorname{Mat}_{m \times n}(\mathbb{R})$ the following inequality holds true:

$$
\|M\|_{\max } \leq\|M\|_{2} \leq \sqrt{m n}\|M\|_{\max } .
$$


Proposition 2.10 Let $r \in[1, \infty]$. The $r$-matrix norm is a sub-multiplicative norm, that is, for each $A \in \operatorname{Mat}_{m \times n}(\mathbb{R}), B \in \operatorname{Mat}_{n \times t}(\mathbb{R})$, one has

$$
\|A B\|_{r} \leq\|A\|_{r}\|B\|_{r}
$$

In particular, for each $A \in \operatorname{Mat}_{m \times n}(\mathbb{R})$ and $v \in \operatorname{Mat}_{n \times 1}(\mathbb{R})$, one has $\|A v\|_{r} \leq\|A\|_{r}\|v\|_{r}$.

We need the following generalization of the Mean Value Theorem for vector valued real functions (see the proof presented in [9]).

Theorem 2.11 Let $U \subseteq \mathbb{R}^{n}$ be a convex open set and let $p \in U$. Let $\phi: U \rightarrow \mathbb{R}^{m}$ be a differentiable vector valued function on $U$. Let $r \in[1, \infty]$. Then, for each $\boldsymbol{x} \in U$, one has

$$
\left\|(\phi(\boldsymbol{x})-\phi(p))^{t}\right\|_{r}<\sup _{0<\nu<1}\left\|\operatorname{Jac}_{\Phi}(p+\nu(\boldsymbol{x}-p))\right\|_{r}\left\|(\boldsymbol{x}-p)^{t}\right\|_{r} .
$$

Finally, let's recall the notion of singular value of a matrix (see [8, Section 2.5.3]).

Theorem-Definition 2.12 (Singular Value Decomposition) Let $A \in \operatorname{Mat}_{m \times n}(\mathbb{R})$ with $m \leq n$. Then there exist orthogonal matrices $U \in \operatorname{Mat}_{m}(\mathbb{R})$ and $V \in \operatorname{Mat}_{n}(\mathbb{R})$ such that

$$
U^{t} A V=\left(\begin{array}{ccccccc}
\sigma_{1} & 0 & \ldots & 0 & 0 & \ldots & 0 \\
0 & \sigma_{2} & \ldots & 0 & 0 & \ldots & 0 \\
& & \vdots & & & \vdots & \\
0 & \ldots & 0 & \sigma_{m} & 0 & \ldots & 0
\end{array}\right) \in \operatorname{Mat}_{m \times n}(\mathbb{R})
$$

where $\sigma_{1} \geq \sigma_{2} \geq \cdots \geq \sigma_{m} \geq 0$ are called the singular values of $A$.

Remark 2.13 The Singular Value Decomposition theorem reveals a great deal about the structure of a matrix. In particular, $r:=\max \left\{j \mid \sigma_{j} \neq 0\right\}$ if and only if $\operatorname{rank}(A)=r$.

Further, there is a connection between 2-matrix norm (see Definition 2.4) and singular values of a matrix $A$. In particular, $\|A\|_{2}=\sigma_{1}$.

\section{$3 \quad$ Necessary non-crossing cell conditions}

In the sequel, let $p=\left(p_{1}, \ldots, p_{n}\right)$ be a point in an open convex set $U \subseteq \mathbb{R}^{n}$, let $r \in[1, \infty]$, and let $\varepsilon$ be a positive real number, also called tolerance threshold. We need the following definition.

Definition 3.1 Let $\varepsilon$ be a positive real number, let $r \in[1, \infty]$, and let $p$ be a point of $U$. The $(r, \varepsilon)$-ball centered at $p$, denoted by $\mathbf{B}_{r}(p, \varepsilon)$, is the closed convex set defined as

$$
\mathbf{B}_{r}(p, \varepsilon)=\left\{\boldsymbol{x} \in \mathbb{R}^{n} \text { such that }\left\|(\boldsymbol{x}-p)^{t}\right\|_{r} \leq \varepsilon\right\},
$$

where the threshold $\varepsilon$ is chosen small enough to assure that $\mathbf{B}_{r}(p, \varepsilon) \subset U$.

The results of this section apply to any subset $\mathbf{C}_{r}(p, \varepsilon)$ of $\mathbf{B}_{r}(p, \varepsilon) \subset U$ such that the interior of both is the same. We refer to $\mathbf{C}_{r}(p, \varepsilon)$ as a $(r, \varepsilon)$-cell centered at $p$.

Let $F=\left(f_{1}, \ldots, f_{m}\right)^{t}$, where $f_{1}, \ldots, f_{m} \in \mathcal{A}(U)$ (see (1)), $m \leq n$. Let $D=$ $\cap_{j=1}^{m} \operatorname{dom}\left(f_{j}\right)$ be the intersection of the domains of all $f_{j}$ 's. Clearly, $U \subseteq D$. 
In this section, we provide a necessary condition on $\|F(p)\|_{r}$ for the zero locus

$$
V(F):=\left\{x \in D \subseteq \mathbb{R}^{n} \mid f_{1}(\boldsymbol{x})=\cdots=f_{m}(\boldsymbol{x})=0\right\}
$$

to cross an $(r, \varepsilon)$-cell $\mathbf{C}_{r}(p, \varepsilon)$ centered at $p$. Let us stress the fact that, from now on, by $V(F)$ we mean the $(m+1)$-ple $\left(V(F), f_{1}, \ldots, f_{m}\right)$ and hence the criterium does depend on the particular equations $f_{j}$ 's we take.

For our purpose, we introduce the function $Q:[1, \infty] \rightarrow[1, \infty]$ defined as

$$
Q(r)=\left\{\begin{array}{cl}
\frac{r}{r-1} & \text { if } r>1 \\
\infty & \text { if } r=1 \\
1 & \text { if } r=\infty
\end{array}\right.
$$

We will also use the quantities $\beta_{r s}^{n \times m}$ as in Proposition 2.7, which gives in this case

$$
\beta_{Q(r) r}^{m \times n}= \begin{cases}n^{\frac{2}{r}-1} & \text { if } r \in[1,2), \\ m^{1-\frac{2}{r}} & \text { if } r \in[2, \infty] .\end{cases}
$$

Our results will also be expressed in terms of the quantity (that depends on the ball $\left.\mathbf{B}_{r}(p, \varepsilon)\right)$

$$
\mathrm{H}^{r}:=\left\|\left(\mathrm{H}_{1}, \ldots, \mathrm{H}_{m}\right)^{t}\right\|_{r},
$$

where $\mathbf{H}_{j}:=\max _{\boldsymbol{x} \in \mathbf{B}_{r}(p, \varepsilon)}\left\{\left\|H_{f_{j}}(\boldsymbol{x})\right\|_{Q(r)}\right\}$. Observe that $\mathbf{B}_{r}(p, \varepsilon)$ is a compact subset of $U$ and that $\left\|H_{f_{j}}(\boldsymbol{x})\right\|_{Q(r)}$ is continuous in $\mathbf{B}_{r}(p, \varepsilon)$. Note also that the $r$-matrix norm is a continuous function of its entries (see [5, Theorem 9.3])). Thus, $\mathrm{H}_{j}$ is well defined.

Theorem 3.2 Let $U \subseteq \mathbb{R}^{n}$ be an open convex set. Let $f_{j}=f_{j}(\boldsymbol{x}): U \rightarrow \mathbb{R}, j=1, \ldots, m$, with $m \leq n$, be real functions in $\mathcal{A}(U)$ and set $F=\left(f_{1}, \ldots, f_{m}\right)^{t}$. Let $p$ be a point of $U$ and let $\mathbf{C}_{r}(p, \varepsilon) \subseteq \mathbf{B}_{r}(p, \varepsilon) \subset U$ be an $(r, \boldsymbol{\varepsilon})$-cell centered at $p$. If

$$
\|F(p)\|_{r}>\left\|\operatorname{Jac}_{F}(p)\right\|_{r} \varepsilon+\frac{1}{2} \beta_{Q(r) r}^{n \times 1} \varepsilon^{2} \mathrm{H}^{r}=: B_{1 r},
$$

then the zero locus $V(F)$ does not cross $\mathbf{C}_{r}(p, \varepsilon)$.

Proof. Suppose by contradiction that the set $V(F)$ crosses the region $\mathbf{C}_{r}(p, \varepsilon)$, that is, suppose that there exists a point $p^{*} \in \mathbf{C}_{r}(p, \varepsilon) \subset U$ belonging to the set $V(F)$. Thus, $f_{j}\left(p^{*}\right)=0$ for each $j=1, \ldots, m$. We apply Taylor's theorem (see Proposition 1.1) to get

$$
0=f_{j}\left(p^{*}\right)=f_{j}(p)+\operatorname{Jac}_{f_{j}}(p)\left(p^{*}-p\right)^{t}+\frac{1}{2}\left(p^{*}-p\right) H_{f_{j}}\left(\xi_{j}\right)\left(p^{*}-p\right)^{t},
$$

where $\xi_{j}$ is a point of the line segment that connects $p$ to $p^{*}, j=1, \ldots, m$, and hence $\xi_{j} \in U$. Hence, letting

$$
R_{1}\left(f_{j}, \xi_{j}\right)=\frac{1}{2}\left(p^{*}-p\right) H_{f_{j}}\left(\xi_{j}\right)\left(p^{*}-p\right)^{t}
$$

whence

$$
\mathbf{0}=F\left(p^{*}\right)=F(p)+\left(\begin{array}{c}
\operatorname{Jac}_{f_{1}}(p) \\
\vdots \\
\operatorname{Jac}_{f_{m}}(p)
\end{array}\right)\left(p^{*}-p\right)^{t}+\left(\begin{array}{c}
R_{1}\left(f_{1}, \xi_{1}\right) \\
\vdots \\
R_{1}\left(f_{m}, \xi_{m}\right)
\end{array}\right) .
$$


Therefore, by the sub-additivity and the sub-multiplicative property of the norms (see Proposition 2.10), we infer that

$$
\begin{aligned}
\|F(p)\|_{r} & \leq\left\|\left(\begin{array}{c}
\operatorname{Jac}_{f_{1}}(p) \\
\vdots \\
\operatorname{Jac}_{f_{m}}(p)
\end{array}\right)\left(p^{*}-p\right)^{t}\right\|_{r}+\left\|\left(\begin{array}{c}
R_{1}\left(f_{1}, \xi_{1}\right) \\
\vdots \\
R_{1}\left(f_{m}, \xi_{m}\right)
\end{array}\right)\right\|_{r} \\
& \leq\left\|\left(\begin{array}{c}
\operatorname{Jac}_{f_{1}}(p) \\
\vdots \\
\operatorname{Jac}_{f_{m}}(p)
\end{array}\right)\right\|_{r}\left\|\left(p^{*}-p\right)^{t}\right\|_{r}+\left\|\left(\begin{array}{c}
R_{1}\left(f_{1}, \xi_{1}\right) \\
\vdots \\
R_{1}\left(f_{m}, \xi_{m}\right)
\end{array}\right)\right\|_{r} \\
& \leq\left\|\left(\begin{array}{c}
\operatorname{Jac}_{f_{1}}(p) \\
\vdots \\
\operatorname{Jac}_{f_{m}}(p)
\end{array}\right)\right\|_{r} \varepsilon+\left\|\left(\begin{array}{c}
R_{1}\left(f_{1}, \xi_{1}\right) \\
\vdots \\
R_{1}\left(f_{m}, \xi_{m}\right)
\end{array}\right)\right\|_{r} .
\end{aligned}
$$

Moreover, using Hölder inequality and the sub-multiplicative property of norms (see propositions 2.2, 2.10), we find

$$
\begin{aligned}
2\left|R_{1}\left(f_{j}, \xi_{j}\right)\right| & =\left|\left(p^{*}-p\right) H_{f_{j}}\left(\xi_{j}\right)\left(p^{*}-p\right)^{t}\right| \\
& \leq\left\|\left(\left(p^{*}-p\right) H_{f_{j}}\left(\xi_{j}\right)\right)^{t}\right\|_{Q(r)}\left\|\left(p^{*}-p\right)^{t}\right\|_{r} \\
& \leq\left\|H_{f_{j}}\left(\xi_{j}\right)^{t}\left(p^{*}-p\right)^{t}\right\|_{Q(r)} \varepsilon \\
& \leq\left\|H_{f_{j}}\left(\xi_{j}\right)^{t}\right\|_{Q(r)}\left\|\left(p^{*}-p\right)^{t}\right\|_{Q(r)} \varepsilon \\
& \leq\left\|H_{f_{j}}\left(\xi_{j}\right)\right\|_{Q(r)} \beta_{Q(r) r}^{n \times 1}\left\|\left(p^{*}-p\right)^{t}\right\|_{r} \varepsilon \\
& \leq \beta_{Q(r) r}^{n \times 1}\left\|H_{f_{j}}\left(\xi_{j}\right)\right\|_{Q(r)} \varepsilon^{2} .
\end{aligned}
$$

Therefore,

$$
\begin{aligned}
\left\|\left(\begin{array}{c}
R_{1}\left(f_{1}, \xi_{1}\right) \\
\vdots \\
R_{1}\left(f_{m}, \xi_{m}\right)
\end{array}\right)\right\|_{r} & \leq \frac{1}{2}\left\|\left(\begin{array}{c}
\beta_{Q(r) r}^{n \times 1}\left\|H_{f_{1}}\left(\xi_{1}\right)\right\|_{Q(r)} \varepsilon^{2} \\
\vdots \\
\beta_{Q(r) r}^{n \times 1}\left\|H_{f_{m}}\left(\xi_{m}\right)\right\|_{Q(r)} \varepsilon^{2}
\end{array}\right)\right\|_{r} \\
& =\frac{1}{2} \beta_{Q(r) r}^{n \times 1} \varepsilon^{2}\left\|\left(\begin{array}{c}
\left\|H_{f_{1}}\left(\xi_{1}\right)\right\|_{Q(r)} \\
\vdots \\
\left\|H_{f_{m}}\left(\xi_{m}\right)\right\|_{Q(r)}
\end{array}\right)\right\|_{r} \\
& \leq \frac{1}{2} \beta_{Q(r) r}^{n \times 1} \varepsilon^{2} \mathrm{H}^{r} .
\end{aligned}
$$

In conclusion, combining relations (10) and (12) we get that $\|F(p)\|_{r} \leq B_{1 r}$, which is a contradiction.

Q.E.D.

Remark 3.3 Combining Theorem 3.2 and expression (6) we get

$$
B_{1 r}= \begin{cases}\left\|\operatorname{Jac}_{F}(p)\right\|_{1} \varepsilon+\frac{1}{2} \varepsilon^{2} \mathrm{H}^{1} & \text { if } r \in[1,2), \\ \left\|\operatorname{Jac}_{F}(p)\right\|_{r} \varepsilon+\frac{1}{2} n^{1-\frac{2}{r}} \varepsilon^{2} \mathrm{H}^{r} & \text { if } r \in[2, \infty] .\end{cases}
$$

Remark 3.4 Notations as in Theorem 3.2. We now consider the special case when $f_{j} \in P$, $j=1, \ldots, m$, and $r=\infty$. Whenever

$$
\left|f_{j}(p)\right|>\left\|\operatorname{Jac}_{f_{j}}(p)\right\|_{\infty} \varepsilon+\frac{n}{2} \max _{\boldsymbol{x} \in \mathbf{B}_{\infty}(p, \varepsilon)}\left\{\left\|H_{f_{j}}(\boldsymbol{x})\right\|_{1}\right\} \varepsilon^{2}, \text { for some } j \in\{1, \ldots, m\},
$$


then $V\left(f_{1}, \ldots, f_{m}\right)$ does not cross $\mathbf{C}_{\infty}(p, \varepsilon)$. This is an obvious consequence of [16, Proposition 2.1] (see also [17]) and hence it provides an alternative necessary non-crossing condition. Let us stress the fact that condition (13) true for each $j=1, \ldots, m$ doesn't imply the assumption $\|F(p)\|_{\infty}>B_{1 \infty}$ of Theorem 3.2 as the Example 3.5 below shows.

In the following examples, in order to compute $B_{1 r}$, one needs to determine $\mathrm{H}^{r}$ (see defining relation (7)). This implies to maximize $\left\|H_{f_{j}}(\boldsymbol{x})\right\|_{Q(r)}$ in the compact ball $\mathbf{B}_{r}(p, \varepsilon)$. For this purpose, we will proceed heuristically by taking finitely many evaluations at random points in the ball and compute the maximum of the $r$-norms at them. We denote by $d_{r}$ the distance under the $r$-norm.

Example 3.5 Let $f_{1}=x^{2}+y^{2}-1, f_{2}=y-2 x^{2}, F=\left(f_{1}, f_{2}\right)^{t}, V(F)=\left\{\boldsymbol{x} \in \mathbb{R}^{2} \mid f_{1}(\boldsymbol{x})=\right.$ $\left.f_{2}(\boldsymbol{x})=0\right\}, p=\left(\frac{1}{2}, \frac{5}{4}\right)$, and take $\varepsilon=0.195$. Some computations are summarized below.

- $\left|f_{1}(p)\right|=\left|\frac{1}{4}+\frac{25}{16}-1\right|=\frac{13}{16} ;$

$\operatorname{Jac}_{f_{1}}(x, y)=(2 x, 2 y)$, so that $\left\|\operatorname{Jac}_{f_{1}}(p)\right\|_{\infty}=\left\|\left(1, \frac{5}{2}\right)\right\|_{\infty}=\frac{7}{2}$;

$H_{f_{1}}(x, y)=\left(\begin{array}{ll}2 & 0 \\ 0 & 2\end{array}\right)$, so $\max _{\boldsymbol{x} \in \mathbf{B}_{\infty}(p, \varepsilon)}\left\{\left\|H_{f_{1}}(\boldsymbol{x})\right\|_{1}\right\}=2$;

$0.8125=\frac{13}{16}=\left|f_{1}(p)\right|>\left\|\operatorname{Jac}_{f_{1}}(p)\right\|_{\infty} \varepsilon+\frac{2}{2} \max _{\boldsymbol{x} \in \mathbf{B}_{\infty}(p, \varepsilon)}\left\{\left\|H_{f_{1}}(\boldsymbol{x})\right\|_{1}\right\} \varepsilon^{2}=\frac{7}{2} \times 0.195+$ $2 \times(0.195)^{2}=0.75855$.

- $\left|f_{2}(p)\right|=\left|\frac{5}{4}-2 \times \frac{1}{4}\right|=\frac{3}{4}$;

$\operatorname{Jac}_{f_{2}}(x, y)=(-4 x, 1)$, so that $\left\|\operatorname{Jac}_{f_{2}}(p)\right\|_{\infty}=\|(-2,1)\|_{\infty}=3$;

$H_{f_{2}}(x, y)=\left(\begin{array}{cc}-4 & 0 \\ 0 & 0\end{array}\right)$, so $\max _{\boldsymbol{x} \in \mathbf{B}_{\infty}(p, \varepsilon)}\left\{\left\|H_{f_{2}}(\boldsymbol{x})\right\|_{1}\right\}=4$;

$0.75=\frac{3}{4}=\left|f_{2}(p)\right|>\left\|\operatorname{Jac}_{f_{2}}(p)\right\|_{\infty} \varepsilon+\frac{2}{2} \max _{\boldsymbol{x} \in \mathbf{B}_{\infty}(p, \varepsilon)}\left\{\left\|H_{f_{2}}(\boldsymbol{x})\right\|_{1}\right\} \varepsilon^{2}=3 \times 0.195+$ $4 \times(0.195)^{2}=0.7371$.

On the other hand,

$$
\|F(p)\|_{\infty}=\left\|\left(\begin{array}{c}
13 / 16 \\
3 / 4
\end{array}\right)\right\|_{\infty}=\frac{13}{16}=0.8125
$$

But,

$$
B_{1 \infty}=\left\|\operatorname{Jac}_{F}(p)\right\|_{\infty} \varepsilon+\frac{1}{2} 2 \varepsilon^{2} \mathrm{H}^{\infty}=3.5 \times 0.195+4 \times(0.195)^{2}=0.8346 .
$$

So, $\|F(p)\|_{\infty}<B_{1 \infty}$. Note that the problem appears because $\left\|\operatorname{Jac}_{F}(p)\right\|_{\infty}$ and $\mathrm{H}^{\infty}$ may not be reached for the same $i \in\{1,2\}$. To have an idea of the picture, we note that

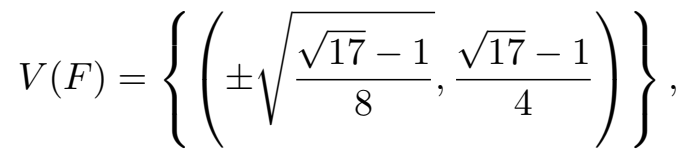

and hence the $\infty$-distance of $p$ to $V(F)$ is $d_{\infty} \approx 0.469$.

For $r \in\{1,2, \infty\}$, we have checked how closed to the distance $d_{r}$ we can take $\varepsilon$ such that the bound $B_{1 r}$ ensures no crossing of the zero locus $V(F)$ with the cell. The output appears in Table 1. The table shows that

$$
\mathbf{B}_{1}(p, 0.29) \cap V(F)=\emptyset, \quad \mathbf{B}_{2}(p, 0.28) \cap V(F)=\emptyset, \quad \mathbf{B}_{\infty}(p, 0.17) \cap V(F)=\emptyset .
$$




\begin{tabular}{c|c|c|c|c}
\hline$r$ & $\varepsilon$ & $d_{r}$ & $B_{1 r}$ & $\|F(p)\|_{r}$ \\
\hline \hline 1 & 0.29 & 0.59 & 1.29 & 1.56 \\
\hline 2 & 0.28 & 0.48 & 0.96 & 1.1 \\
\hline$\infty$ & 0.17 & 0.46 & 0.7 & 0.81 \\
\hline
\end{tabular}

Table 1: Results of the executions corresponding to Example 3.5

Example 3.6 In this example we consider the polynomials $f_{1}=x^{2}+y^{2}+z^{2}-4, f_{2}=(x-$ $1)^{2}+y^{2}-1$, and $F=\left(f_{1}, f_{2}\right)^{t}$; note that the zero locus $V(F)=\left\{\boldsymbol{x} \in \mathbb{R}^{3} \mid f_{1}(\boldsymbol{x})=f_{2}(\boldsymbol{x})=\right.$ $0\}$ is classically known as Viviani Curve. In addition, we take the point $p=(1,0,0)$. The distance of $p$ to the Viviani curve is $d_{\infty} \approx 0.73, d_{1} \approx 1$, and $d_{2} \approx 1$, where $d_{r}$ means the distance under the $r$-norm. In this situation, we have analyzed how big $\varepsilon$ can be taken so that $B_{1 r}<\|F(p)\|_{r}$. The results for $r \in\{1,2, \infty\}$ appear in Table 2. The table shows that

$$
\mathbf{B}_{1}(p, 0.9) \cap V(F)=\emptyset, \quad \mathbf{B}_{2}(p, 0.9) \cap V(F)=\emptyset, \quad \mathbf{B}_{\infty}(p, 0.63) \cap V(F)=\emptyset .
$$

\begin{tabular}{c|c|c|c|c}
\hline$r$ & $\varepsilon$ & $d_{r}$ & $B_{1 r}$ & $\|F(p)\|_{r}$ \\
\hline \hline 1 & 0.9 & 1 & 3.42 & 4 \\
\hline 2 & 0.9 & 1 & 2.95 & 3.16 \\
\hline$\infty$ & 0.63 & 0.73 & 2.46 & 3 \\
\hline
\end{tabular}

Table 2: Results of the executions corresponding to Example 3.6

Example 3.7 In this example we consider the real functions $z=f_{1}(x, y), z=f_{2}(x, y)$, where

$$
f_{1}(x, y)=\frac{e^{x}}{x^{2}+y^{2}+1}, \quad f_{2}(x, y)=\frac{e^{y}}{x^{2}+y^{2}+1} .
$$

Let $F=\left(z-f_{1}(x, y), z-f_{2}(x, y)\right)^{t}$. We consider in $\mathbb{R}^{3}$ the zero locus $V(F)=\{(x, y, z) \in$ $\left.\mathbb{R}^{3} \mid z-f_{1}(x, y)=z-f_{2}(x, y)=0\right\}$ Note that $V(F)$ can be parametrized as $\left(t, t, e^{t} /(1+\right.$ $\left.2 t^{2}\right)$ ).

In addition, we take the point $p=(0,0,0)$. The distances of $p$ to $V(F)$ are $d_{\infty} \approx 0.453$, $d_{1} \approx 1$, and $d_{2} \approx 0.75$. In this situation, we have analyzed how big $\varepsilon$ can be taken so that $B_{1 r}<\|F(p)\|_{r}$. The results for $r \in\{1,2, \infty\}$ appear in Table 3 . The table gives the non-crossing conditions

$$
\mathbf{B}_{1}(p, 0.7) \cap V(F)=\emptyset, \quad \mathbf{B}_{2}(p, 0.65) \cap V(F)=\emptyset, \quad \mathbf{B}_{\infty}(p, 0.35) \cap V(F)=\emptyset .
$$

\section{Sufficient crossing cell conditions}

Keeping the notation as in previous sections, let $F=\left(f_{1}, \ldots, f_{m}\right)^{t}$, where $f_{1}, \ldots, f_{m} \in$ $\mathcal{A}(U)$, see (1), where $U \subseteq \mathbb{R}^{n}$ is a convex open set, with $m \leq n$. Let $D \subseteq \mathbb{R}^{n}$ be the 


\begin{tabular}{c|c|c|c|c}
\hline$r$ & $\varepsilon$ & $d_{r}$ & $B_{1 r}$ & $\|F(p)\|_{r}$ \\
\hline \hline 1 & 0.7 & 1 & 1.71 & 2 \\
\hline 2 & 0.65 & 0.75 & 1.31 & 1.41 \\
\hline$\infty$ & 0.35 & 0.45 & 0.78 & 1 \\
\hline
\end{tabular}

Table 3: Results of the executions corresponding to Example 3.7

intersection of the domains of $f_{1}, \ldots, f_{m}$. In this section we provide sufficient numerical conditions for the zero locus

$$
V(F):=\left\{\boldsymbol{x} \in D \subseteq \mathbb{R}^{n} \mid f_{1}(\boldsymbol{x})=\cdots=f_{m}(\boldsymbol{x})=0\right\}
$$

to cross a bounded region containing a given point of $U$. For this purpose, we need to use the Moore-Penrose pseudo-inverse of matrices with entries in $\mathcal{A}(U)$ (see [8, §5.5.4]). So, first, we prove that the pseudo-inverse exists. Let $\mathbb{F}$ be the quotient field of the integral domain $\mathcal{A}(U)$. An element $g$ in $\mathbb{F}$, can be expressed as $a / b$ with $a, b \in \mathcal{A}(U)$, and it is the zero-element of $\mathbb{F}$ iff $a(\boldsymbol{x})=0$ for all $\boldsymbol{x} \in U$ or, equivalently, iff $a(\boldsymbol{x})=0$ for all $\boldsymbol{x}$ in a non-empty open subset of $U$ (see Principle of Analytic Continuation in [4, p. 122). Now we consider the ring $\operatorname{Mat}_{m \times n}(\mathbb{F})$ of the $m \times n$ matrices with entries in $\mathbb{F}$. Then, for $M \in \operatorname{Mat}_{m \times n}(\mathbb{F})$ we prove that the Moore-Penrose pseudo-inverse of $M$, taking as involutory automorphism the identity, exists; we denote the pseudo-inverse as $M^{\dagger}$. More precisely, we prove the following lemma.

Lemma 4.1 For every matrix $M$ in $\operatorname{Mat}_{m \times n}(\mathbb{F})$ there exists $M^{\dagger}$, and $M^{\dagger} \in \operatorname{Mat}_{n \times m}(\mathbb{F})$.

Proof. We will prove the lemma by using Theorem 4 in [14]. Let us denote the zero of $\mathbb{F}$ as $0_{\mathbb{F}}$. Let us see that if $g_{1}, \ldots, g_{\ell} \in \mathbb{F}$ satisfy the condition $g:=g_{1}^{2}+\cdots+g_{\ell}^{2}=0_{\mathbb{F}}$, then $g_{1}=\cdots=g_{\ell}=0_{\mathbb{F}}$. Each $g_{i}$ can be expressed as $g_{i}=a_{i} / b_{i}$, where $a_{i}, b_{i} \in \mathcal{A}(U)$ and $b_{i} \neq 0$. Let $B_{i}=\prod_{k=1, k \neq i}^{\ell} b_{k}^{2}$ and let $B=\prod_{i=1}^{\ell} b_{i}^{2}$. Then, $g=\left(a_{1}^{2} B_{1}+\cdots+a_{\ell}^{2} B_{\ell}\right) / B$, and $a_{1}^{2} B_{1}+\cdots+a_{\ell}^{2} B_{\ell}=0_{\mathbb{F}}$. On the other hand, $B \neq 0_{\mathbb{F}}$. Thus, there exists $p \in U$ such that $B(p)>0$. Since $B$ is continuous in $U$, there exists an open subset $\Omega \subset U$ where $B$ does not vanish and hence $B_{1}, \ldots, B_{\ell}$ do not vanish either; indeed they take positive values. Now, we observe that for all $p \in \Omega$ it holds that $a_{1}^{2}(p) B_{1}(p)+\cdots+a_{\ell}^{2}(p) B_{\ell}(p)=0$. Therefore, $a_{i}(p)=0$ for all $i$ and for all $p \in \Omega$. Thus, $a_{1}=\cdots=a_{\ell}=0_{\mathbb{F}}$. In this situation, Theorem 4 in [14] implies that $M^{\dagger}$ exists. The fact that $M^{\dagger}$ is over $\mathbb{F}$ follows from Theorem 3 in [10], p. 116.

Q.E.D.

Remark 4.2 We observe that if we replace $\mathcal{A}(U)$ by the $\operatorname{ring} \mathcal{C}^{\infty}(U)$ of the functions of class infinity in $U$, then we do not have an integral domain, so that matrices with entries in $\mathcal{C}^{\infty}(U)$ may not have Moore-Penrose inverse. For instance, let us consider the functions $f, g \in \mathcal{C}^{\infty}(\mathbb{R})$ defined as

$$
f(x)=\left\{\begin{array}{ll}
0 & \text { if } x \leq 0 \\
e^{-1 / x^{2}} & \text { if } x>0
\end{array}, g(x)= \begin{cases}e^{-1 / x^{2}} & \text { if } x \leq 0 \\
0 & \text { if } x>0\end{cases}\right.
$$

as well as the matrix $M=(f g)$. Then $M M^{t}=\left(f^{2}+g^{2}\right)$. So, $\operatorname{rank}(M)=1$ and $\operatorname{rank}\left(M M^{t}\right)=1$. But

$$
M^{t} M=\left(\begin{array}{cc}
f^{2} & 0 \\
0 & g^{2}
\end{array}\right)
$$


has rank 2. Therefore, by Theorem 3 in [10, p. 116, we conclude that $M^{\dagger}$ does not exist. Note that the problem comes from the fact that $f, g$ are zero divisors of the $\operatorname{ring} \mathcal{C}^{\infty}(\mathbb{R})$.

We fix $r \in[1, \infty]$, and for any positive real number $R<\varepsilon$, we will consider the open $r$-ball

$$
\mathcal{D}_{r}(p, R):=\left\{\boldsymbol{x} \in \mathbb{R}^{n} \text { such that }\left\|(\boldsymbol{x}-p)^{t}\right\|_{r}<R\right\} .
$$

Taking the closure, one has $\overline{\mathcal{D}_{r}(p, R)}=\mathbf{B}_{r}(p, R) \subseteq \mathbf{C}_{r}(p, \varepsilon) \subset \mathbf{B}_{r}(p, \varepsilon)$.

We start with the following technical results.

Lemma 4.3 Let $M(\boldsymbol{x})$ be an $m \times n$ matrix whose entries belong to $\mathcal{A}(U)$, and let $p$ in $U$ be such that $\operatorname{rank}(M(p))=: \rho>0$. Then there exists $0<R$ such that, for every $q \in \mathbf{B}_{r}(p, R)$, $\operatorname{rank}(M(q))=\rho$.

Proof. Let $N(\boldsymbol{x})$ be one of the $\rho \times \rho$ submatrices of $M(\boldsymbol{x})$ such that $\operatorname{det}(N(p)) \neq 0$. Let $g(\boldsymbol{x})=\operatorname{det}(N(\boldsymbol{x}))$. Since the entries of $M$ are in $\mathcal{A}(U)$, then $g(\boldsymbol{x}) \in \mathcal{A}(U)$, and, in particular, $g$ is continuous in $U$. Therefore there exists an open 2-ball $\Omega$ centered at $p$ where the sign of $g(\boldsymbol{x})$ is the sign of $g(p)$ for $\boldsymbol{x} \in \Omega$. By taking $R$ sufficiently small such that $\mathbf{B}_{r}(p, R) \subset \Omega$, we are done.

Q.E.D.

Lemma 4.4 Let $M(\boldsymbol{x})$ be an $m \times n$ matrix whose entries belong to $\mathcal{A}(U)$, and let $p$ in $U$ be such that, for every $q \in \mathbf{B}_{r}(p, R), M(q)$ is full rank. Then the entries of $M^{\dagger}(\boldsymbol{x})$ are analytic in $\mathcal{D}_{r}(p, R)$.

Proof. Let $\rho:=\min \{m, n\}=m$. Let $N(\boldsymbol{x})$ be one of the $\rho \times \rho$ submatrices of $M(\boldsymbol{x})$ such that $\operatorname{det}(N(p)) \neq 0$. Let $g(\boldsymbol{x})=\operatorname{det}(N(\boldsymbol{x}))$. Therefore, $M(\boldsymbol{x})$ is full rank for every $\boldsymbol{x} \in \mathbf{B}_{r}(p, R)$, and $g(\boldsymbol{x}) \neq 0$ for all $\boldsymbol{x}$ in this set. Now, we consider the integral domain $\mathcal{A}\left(\mathcal{D}_{r}(p, R)\right)$ as well as its field of fractions $\mathbb{L}$. Then, $M \in$ Mat $_{m \times n}(\mathbb{L})$ with rank $m$ since $g \neq 0_{\mathbb{L}}$. Thus, by Theorem 3 in [10], p. 116, and since by Lemma 4.1 the pseudo-inverse $M^{\dagger}(\boldsymbol{x})$ exists, $M^{\dagger}(\boldsymbol{x})$ can be expressed as

$$
M^{\dagger}(\boldsymbol{x}):=M(\boldsymbol{x})^{t}\left(M(\boldsymbol{x}) M^{t}(\boldsymbol{x})\right)^{-1} .
$$

Therefore, the denominator of each entries of $M^{\dagger}$ can be taken as $h(\boldsymbol{x})=\operatorname{det}\left(M(\boldsymbol{x}) M^{t}(\boldsymbol{x})\right)$. So, it only remains to check that $h(\boldsymbol{x}) \neq 0$ for all $\boldsymbol{x} \in \mathcal{D}_{r}(p, R)$. Indeed, let $q \in \mathcal{D}_{r}(p, R)$. Then $\operatorname{rank}(M(q))=m$, then $\operatorname{rank}\left(M(q) M^{t}(q)\right)=m$ so that the matrices are real. Thus, $h(q) \neq 0$.

Q.E.D.

Lemma 4.5 Let $U \subseteq \mathbb{R}^{n}$ be an open convex set, and let $f_{j}=f_{j}(\boldsymbol{x}) \in \mathcal{A}(U), j=1, \ldots, m$, $m \leq n$. Let $F=\left(f_{1}, \ldots, f_{m}\right)^{t}$. Assume that the Hessian $H_{F}(\boldsymbol{x})$ is not the zero matrix. Let $p \in U$ be such that $\rho:=\operatorname{rank}\left(\operatorname{Jac}_{F}(p)\right)>0$ and let $\sigma_{\rho}\left(\operatorname{Jac}_{F}(p)\right)$ be the $\rho$-th singular value of the matrix $\operatorname{Jac}_{F}(p)$. Set

$$
0<R<\min \left\{\varepsilon, \frac{\sigma_{\rho}\left(\operatorname{Jac}_{F}(p)\right)}{\sqrt{m n} \mathrm{H}^{\infty}}\right\}=: \bar{R} .
$$

Then, for every $q \in \mathbf{B}_{r}(p, R)$ and $r \in[1, \infty]$, it holds that $\operatorname{rank}\left(\operatorname{Jac}_{F}(q)\right)=\operatorname{rank}\left(\operatorname{Jac}_{F}(p)\right)$. 
Proof. Since $\operatorname{rank}(\operatorname{Jac}(\boldsymbol{x}))=\rho$ then $\sigma_{\rho}:=\sigma_{\rho}\left(\operatorname{Jac}_{F}(p)\right) \neq 0$ (see Remark 2.13). Thus, by the Eckart-Young theorem (see [8, p. 72]), there exists a matrix $A \in \operatorname{Mat}_{m \times n}(\mathbb{R})$ such that $\operatorname{rank}(A)=\rho-1$ and

$$
\left\|A-\operatorname{Jac}_{F}(p)\right\|_{2}=\min _{\substack{M \in \operatorname{Mat}_{m \times n}(\mathbb{R}) \\ \operatorname{rank}(M) \leq \rho-1}}\left\{\left\|M-\operatorname{Jac}_{F}(p)\right\|_{2}\right\}=\sigma_{\rho} .
$$

In particular, for each $M \in \operatorname{Mat}_{m \times n}(\mathbb{R})$ such that $\left\|M-\operatorname{Jac}_{F}(p)\right\|_{2}<\sigma_{\rho}$, then $\operatorname{rank}(M)=$ $\rho$.

For the next part of the proof, we take $R^{*}$ such that $R<R^{*}<\bar{R}$. Now, we apply the Mean Value Theorem 2.11 to the differentiable vector valued real function $\phi:=\left(\operatorname{Jac}_{F}^{(\mathbf{v})}(\boldsymbol{x})\right)^{t}: \mathbb{R}^{n} \rightarrow \mathbb{R}^{m n}$. Then, recalling that $\beta_{\infty r}^{n \times 1}=1$, for each $\boldsymbol{x} \in \mathcal{D}_{r}\left(p, R^{*}\right) \subset$ $\mathbf{B}_{r}\left(p, R^{*}\right) \subset \mathcal{D}_{r}(p, \varepsilon) \subset \mathcal{D}_{\infty}(p, \varepsilon)$, we have

$$
\begin{aligned}
\left\|\operatorname{Jac}_{F}^{(\mathbf{v})}(\boldsymbol{x})-\operatorname{Jac}_{F}^{(\mathbf{v})}(p)\right\|_{\infty} & <\sup _{0<\nu<1}\left\|\left(\begin{array}{c}
H_{f_{1}}(p+\nu(\boldsymbol{x}-p)) \\
\vdots \\
H_{f_{m}}(p+\nu(\boldsymbol{x}-p))
\end{array}\right)\right\|_{\infty}\left\|(\boldsymbol{x}-p)^{t}\right\|_{\infty} \\
& \leq \sup _{0<\nu<1}\left\|\left(\begin{array}{c}
H_{f_{1}}(p+\nu(\boldsymbol{x}-p)) \\
\vdots \\
H_{f_{m}}(p+\nu(\boldsymbol{x}-p))
\end{array}\right)\right\|_{\infty}\left\|(\boldsymbol{x}-p)^{t}\right\|_{r} \\
& <\sup _{0<\nu<1}\left\|\left(\begin{array}{c}
H_{f_{1}}(p+\nu(\boldsymbol{x}-p)) \\
\vdots \\
H_{f_{m}}(p+\nu(\boldsymbol{x}-p))
\end{array}\right)\right\|_{\infty} R^{*} \\
& =\sup _{0<\nu<1}\left\{\max _{j=1, \ldots, m}\left\|H_{f_{j}}(p+\nu(\boldsymbol{x}-p))\right\|_{\infty}\right\} R^{*} \\
& =\max _{j=1, \ldots, m}\left\{\sup _{0<\nu<1}\left\|H_{f_{j}}(p+\nu(\boldsymbol{x}-p))\right\|_{\infty}\right\} R^{*} \\
& \leq \max _{j=1, \ldots, m}\left\{\sup _{\boldsymbol{x} \in \mathbf{B}\left(p, R^{*}\right)}\left\|H_{f_{j}}(\boldsymbol{x})\right\|_{1}\right\} R^{*} \\
& \leq \max _{j=1, \ldots, m}\left\{\sup _{\boldsymbol{x} \in \mathbf{B}_{\infty}(p, \varepsilon)}\left\|H_{f_{j}}(\boldsymbol{x})\right\|_{1}\right\} R^{*} \\
& =\mathrm{H}^{\infty} R^{*} .
\end{aligned}
$$

On the other hand,

$$
\left\|\operatorname{Jac}_{F}^{(\mathbf{v})}(\boldsymbol{x})-\operatorname{Jac}_{F}^{(\mathbf{v})}(p)\right\|_{\infty}=\left\|\operatorname{Jac}_{F}(\boldsymbol{x})-\operatorname{Jac}_{F}(p)\right\|_{\max } \geq \frac{1}{\sqrt{m n}}\left\|\operatorname{Jac}_{F}(\boldsymbol{x})-\operatorname{Jac}_{F}(p)\right\|_{2} .
$$

By combining with inequality (17), we then find

$$
\frac{1}{\sqrt{m n}}\left\|\operatorname{Jac}_{F}(\boldsymbol{x})-\operatorname{Jac}_{F}(p)\right\|_{2}<\mathrm{H}^{\infty} R^{*} .
$$

Whence, by the assumptions on $R^{*}<\bar{R}$,

$$
\left\|\operatorname{Jac}_{F}(\boldsymbol{x})-\operatorname{Jac}_{F}(p)\right\|_{2}<\sqrt{m n} \mathrm{H}^{\infty} \bar{R} \leq \sigma_{\rho} .
$$


By the consequence noted above of Eckart-Young theorem (see expression (16p), it then follows that $\operatorname{rank}\left(\operatorname{Jac}_{F}(\boldsymbol{x})\right)=\rho$ for each $\boldsymbol{x} \in \mathcal{D}_{r}\left(p, R^{*}\right)$. Now, since $\mathcal{D}_{r}(p, R) \subset \mathbf{B}_{r}(p, R) \subset$ $\mathcal{D}_{r}\left(p, R^{*}\right)$, the result follows.

In the sequel, we will take $p \in U$ such that $\operatorname{rank}\left(\operatorname{Jac}_{F}(p)\right)=m$, and $R$ satisfies the hypotheses of Lemma 4.5. In addition, we assume without loss of generality that $R$ is sufficiently small to ensure that the entries of $\operatorname{Jac}_{F}^{\dagger}(\boldsymbol{x})$ are continuous in $\mathbf{B}_{r}(p, R)$, see Lemma 4.4. Now, we define the quantity

$$
\mathrm{J}^{r}:=\max _{\boldsymbol{x} \in \mathbf{B}_{r}(p, R)}\left\{\left\|\operatorname{Jac}_{F}^{\dagger}(\boldsymbol{x})\right\|_{r}\right\}
$$

Note that the existence of $\mathrm{J}^{r}$ is guaranteed by the classical Weierstrass theorem applied to the continuous function $\left\|\operatorname{Jac}_{F}^{\dagger}(\boldsymbol{x})\right\|_{r}$ on the compact set $\mathbf{B}_{r}(p, R)$. The continuity of the real-valued function $\left\|\operatorname{Jac}_{F}^{\dagger}(\boldsymbol{x})\right\|_{r}: \mathbf{B}_{r}(p, R) \rightarrow \mathbb{R}$ derives from the fact that the entries of the Moore-Penrose inverse have been taken continuous and recalling that the $r$-matrix norm is a continuous function of its entries (see [5, Theorem 9.3]).

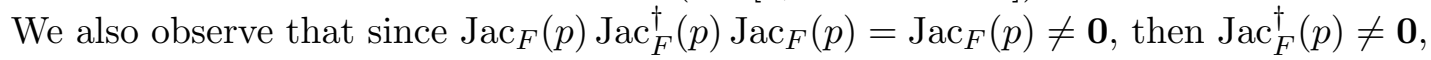
and so $\mathrm{J}^{r} \neq 0$.

Theorem 4.6 Let $U \subseteq \mathbb{R}^{n}$ be an open convex set, and let $f_{j}=f_{j}(\boldsymbol{x}) \in \mathcal{A}(U), j=$ $1, \ldots, m, m \leq n$, let $F=\left(f_{1}, \ldots, f_{m}\right)^{t}$. Assume that the Hessian $H_{F}(\boldsymbol{x})$ is not the zero matrix. Let $p \in U$ such that $\operatorname{rank}\left(\operatorname{Jac}_{F}(p)\right)=m$, and let $R$ be a positive real number such that

$$
R<\min \left\{\varepsilon, \frac{\sigma_{m}\left(\operatorname{Jac}_{F}(p)\right)}{\sqrt{m n} \mathrm{H}^{\infty}}\right\}
$$

If

$$
\|F(p)\|_{r}<\frac{2 R}{\mathrm{~J}^{r}\left(2+\beta_{Q(r) r}^{m \times 1} \beta_{Q(r) r}^{n \times m} R \mathrm{~J}^{r} \mathrm{H}^{r}\right)}=: B_{2 r},
$$

then the zero locus $V(F)=\left\{f_{1}=\cdots=f_{m}=0\right\}$ crosses $\mathbf{C}_{r}(p, \varepsilon)$.

Proof. First, note that if $\|F(p)\|_{r}=0$ then $F(p)=\mathbf{0}$, and hence $p \in V(F)$. So, in this case, the result is trivial. In the rest of the proof we assume that $\|F(p)\|_{r}>0$.

By Lemma 4.5 we know that $\operatorname{Jac}_{F}(\boldsymbol{x})$ is of full rank $m$ for each $\boldsymbol{x} \in \mathcal{D}_{r}(p, R)$. Moreover, since $R<\varepsilon$, one has $\mathcal{D}_{r}(p, R) \subset \mathbf{C}_{r}(p, \varepsilon)$. We now construct a sequence of points $\left\{p_{k}\right\}_{k \in \mathbb{N}}$ as follows. We let $p_{0}=p$ and, for each $k \geq 0$, we define

$$
s_{k}:=-\operatorname{Jac}_{F}^{\dagger}\left(p_{k}\right) F\left(p_{k}\right) \in \operatorname{Mat}_{n \times 1}(\mathbb{R}) \text { and } p_{k+1}:=p_{k}+s_{k}{ }^{t} \in \operatorname{Mat}_{1 \times n}(\mathbb{R}) .
$$

As in the proof of Proposition 3.2 in [16], the construction of the points $p_{k}$ 's draws back to the Normal Flow Algorithm (see [20]), an iterative method often used in homotopy and continuation problems. Obviously, $p=p_{0} \in \mathcal{D}_{r}(p, R)$. We prove by induction that the points $p_{k}$ 's all lie in $\mathcal{D}_{r}(p, R)$, and satisfy the inequality

$$
\left\|F\left(p_{k}\right)\right\|_{r}<\left\|F\left(p_{k-1}\right)\right\|_{r}, \quad k \geq 1 .
$$

Step I (The $k=1$ case). From the definition of $s_{0}$ and $J^{r}$ (see 19 ) we have

$$
\left\|s_{0}\right\|_{r}=\left\|\operatorname{Jac}_{F}^{\dagger}(p) F(p)\right\|_{r} \leq\left\|\operatorname{Jac}_{F}^{\dagger}(p)\right\|_{r}\|F(p)\|_{r} \leq J^{r}\|F(p)\|_{r} .
$$


By the assumption it follows that $\|F(p)\|_{r}<B_{2 r}<\frac{R}{\mathrm{~J}^{r}}$, so that the above inequality yields $\left\|s_{0}\right\|_{r}<R$, showing that $p_{1} \in \mathcal{D}_{r}(p, R)$. Moreover, we have (we use the same notation as in the proof of Theorem 3.2 .

$$
\begin{aligned}
F\left(p_{1}\right) & =F(p)+\operatorname{Jac}_{F}(p)\left(p_{1}-p\right)^{t}+\left(\begin{array}{c}
R_{1}\left(f_{1}, \xi_{1}\right) \\
\vdots \\
R_{1}\left(f_{m}, \xi_{m}\right)
\end{array}\right) \\
& =F(p)+\operatorname{Jac}_{F}(p) s_{0}+\left(\begin{array}{c}
R_{1}\left(f_{1}, \xi_{1}\right) \\
\vdots \\
R_{1}\left(f_{m}, \xi_{m}\right)
\end{array}\right) \\
& =F(p)+\operatorname{Jac}_{F}(p)\left(-\operatorname{Jac}_{F}^{\dagger}(p) F(p)\right)+\left(\begin{array}{c}
R_{1}\left(f_{1}, \xi_{1}\right) \\
\vdots \\
R_{1}\left(f_{m}, \xi_{m}\right)
\end{array}\right) \\
& \left.=\frac{1}{2}\left(\begin{array}{c}
s_{0}^{t} H_{f_{1}}\left(\xi_{1}\right) s_{0} \\
\vdots \\
s_{0}^{t} H_{f_{m}}\left(\xi_{m}\right) s_{0}
\end{array}\right) \begin{array}{c}
F(p)^{t} \operatorname{Jac}_{F}^{\dagger}(p)^{t} H_{f_{1}}\left(\xi_{1}\right) \operatorname{Jac}_{F}^{\dagger}(p) F(p) \\
\vdots \\
F(p)^{t} \operatorname{Jac}_{F}^{\dagger}(p)^{t} H_{f_{m}}\left(\xi_{m}\right) \operatorname{Jac}_{F}^{\dagger}(p) F(p)
\end{array}\right)
\end{aligned}
$$

where $\xi_{j}$ is a point of the line segment that connects $p$ to $p_{1}$, and $R_{1}\left(f_{j}, \xi_{j}\right)$ is defined as in $(9), j=1, \ldots, m$. We now bound each entry of the matrix above

$$
\begin{aligned}
\left|F(p)^{t} \operatorname{Jac}_{F}^{\dagger}(p)^{t} H_{f_{j}}\left(\xi_{j}\right) \operatorname{Jac}_{F}^{\dagger}(p) F(p)\right| & \leq\left\|\left(F(p)^{t} \operatorname{Jac}_{F}^{\dagger}(p)^{t} H_{f_{j}}\left(\xi_{j}\right)\right)^{t}\right\|_{Q(r)}\left\|\operatorname{Jac}_{F}^{\dagger}(p) F(p)\right\|_{r} \\
& \leq\|F(p)\|_{Q(r)}\left\|\operatorname{Jac}_{F}^{\dagger}(p)\right\|_{Q(r)}\left\|H_{f_{j}}\left(\xi_{j}\right)\right\|_{Q(r)}\left\|\operatorname{Jac}_{F}^{\dagger}(p)\right\|_{r}\|F(p)\|_{r} \\
& \leq\|F(p)\|_{Q(r)} \beta_{Q(r) r}^{n \times m}\left\|H_{f_{j}}\left(\xi_{j}\right)\right\|_{Q(r)}\left\|\operatorname{Jac}_{F}^{\dagger}(p)\right\|_{r}^{2}\|F(p)\|_{r} \\
& \leq\|F(p)\|_{Q(r)} \beta_{Q(r) r}^{n \times m}\left(\mathrm{~J}^{r}\right)^{2} \mathrm{H}_{\mathrm{j}}\|F(p)\|_{r} \\
& \leq \beta_{Q(r) r}^{m \times 1}\|F(p)\|_{r} \beta_{Q(r) r}^{n \times m}\left(\mathrm{~J}^{r}\right)^{2} \mathrm{H}_{\mathrm{j}}\|F(p)\|_{r} \\
& =\beta_{Q(r) r}^{m \times 1} \beta_{Q(r) r}^{n \times m}\left(\mathrm{~J}^{r}\right)^{2} \mathrm{H}_{\mathrm{j}}\|F(p)\|_{r}^{2} .
\end{aligned}
$$

Therefore

$$
\begin{aligned}
\left\|F\left(p_{1}\right)\right\|_{r} & =\frac{1}{2}\left\|\left(\begin{array}{c}
F(p)^{t} \operatorname{Jac}_{F}^{\dagger}(p)^{t} H_{f_{1}}\left(\xi_{1}\right) \operatorname{Jac}_{F}^{\dagger}(p) F(p) \\
\vdots \\
F(p)^{t} \operatorname{Jac}_{F}^{\dagger}(p)^{t} H_{f_{m}}\left(\xi_{m}\right) \operatorname{Jac}_{F}^{\dagger}(p) F(p)
\end{array}\right)\right\|_{r} \\
& \leq \frac{1}{2}\left\|\beta_{Q(r) r}^{m \times 1} \beta_{Q(r) r}^{n \times m}\right\| F(p)\left\|_{r}^{2}\left(\mathrm{~J}^{r}\right)^{2}\left(\mathrm{H}_{1}, \ldots, \mathrm{H}_{m}\right)^{t}\right\|_{r} \\
& =\frac{1}{2} \beta_{Q(r) r}^{m \times 1} \beta_{Q(r) r}^{n \times m}\|F(p)\|_{r}^{2}\left(\mathrm{~J}^{r}\right)^{2}\left\|\left(\mathrm{H}_{1}, \ldots, \mathrm{H}_{m}\right)^{t}\right\|_{r} \\
& =\frac{1}{2} \beta_{Q(r) r}^{m \times 1} \beta_{Q(r) r}^{n \times m}\|F(p)\|_{r}^{2}\left(\mathrm{~J}^{r}\right)^{2} \mathrm{H}^{r} \\
& <\frac{1}{2} \beta_{Q(r) r}^{m \times 1} \beta_{Q(r) r}^{n \times m} B_{2 r}\left(\mathrm{~J}^{r}\right)^{2} \mathrm{H}^{r}\|F(p)\|_{r} .
\end{aligned}
$$


Finally, since

$$
B_{2 r}=\frac{2}{J^{r}\left(\frac{2}{R}+\beta_{Q(r) r}^{m \times 1} \beta_{Q(r) r}^{n \times m} \mathrm{~J}^{r} \mathrm{H}^{r}\right)}<\frac{2}{\beta_{Q(r) r}^{m \times 1} \beta_{Q(r) r}^{n \times m}\left(\mathrm{~J}^{r}\right)^{2} \mathrm{H}^{r}},
$$

one concludes that $\left\|F\left(p_{1}\right)\right\|_{r}<\|F(p)\|_{r}$.

Step II (The inductive step). Suppose that the points $p, p_{1}, \ldots, p_{k}$ of the sequence all lie in $\mathcal{D}_{r}(p, R)$, and that $0<\left\|F\left(p_{k}\right)\right\|_{r}<\left\|F\left(p_{k-1}\right)\right\|_{r}<\cdots<\|F(p)\|_{r}$. Hence, in particular, the points $p, p_{1}, \ldots, p_{k}$ are all distinct, so that, by definition, $\left\|s_{i-1}\right\|_{r} \neq 0$ for $i=1, \ldots, k$.

First, we show that $p_{k+1} \in \mathcal{D}_{r}(p, R)$. Arguing as in Step I, we have, for $i=1, \ldots, k$,

$$
F\left(p_{i}\right)=F\left(p_{i-1}\right)+\operatorname{Jac}_{F}\left(p_{i-1}\right) s_{i-1}+\left(\begin{array}{c}
\frac{1}{2} s_{i-1}^{t} H_{f_{1}}\left(\xi_{1}\right) s_{i-1} \\
\vdots \\
\frac{1}{2} s_{i-1}^{t} H_{f_{m}}\left(\xi_{m}\right) s_{i-1}
\end{array}\right) .
$$

for some point $\xi_{j}=\xi_{j}^{(i)}$ (depending on $i$ ) of the line segment that connects $p_{i-1}$ to $p_{i}$, $j=1, \ldots, m$. Moreover, since $s_{i-1}=-\operatorname{Jac}_{F}^{\dagger}\left(p_{i-1}\right) F\left(p_{i-1}\right)$ and $\operatorname{Jac}_{F}(\boldsymbol{x}) \operatorname{Jac}_{F}^{\dagger}(\boldsymbol{x})=\mathbf{I}_{m}$, one has $\operatorname{Jac}_{F}\left(p_{i-1}\right) s_{i-1}=-F\left(p_{i-1}\right)$. So,

$$
\left\|F\left(p_{i}\right)\right\|_{r}=\left\|\left(\begin{array}{c}
\frac{1}{2} s_{i-1}^{t} H_{f_{1}}\left(\xi_{1}\right) s_{i-1} \\
\vdots \\
\frac{1}{2} s_{i-1}^{t} H_{f_{m}}\left(\xi_{m}\right) s_{i-1}
\end{array}\right)\right\|_{r} .
$$

Let us bound each entry of the matrix as follows. Since

$$
\left\|s_{i-1}\right\|_{Q(r)} \leq \beta_{Q(r) r}^{n \times 1}\left\|s_{i-1}\right\|_{r} \leq \beta_{Q(r) r}^{n \times m}\left\|s_{i-1}\right\|_{r},
$$

(see Corollary $2.8(2))$ we get that

$$
\begin{aligned}
\left|s_{i-1}^{t} H_{f_{j}}\left(\xi_{j}\right) s_{i-1}\right| & \leq\left\|H_{f_{j}}\left(\xi_{j}\right) s_{i-1}\right\|_{Q(r)}\left\|s_{i-1}\right\|_{r} \\
& \leq\left\|H_{f_{j}}\left(\xi_{j}\right)\right\|_{Q(r)}\left\|s_{i-1}\right\|_{Q(r)}\left\|s_{i-1}\right\|_{r} \\
& \leq \beta_{Q(r) r}^{n \times m}\left\|H_{f_{j}}\left(\xi_{j}\right)\right\|_{Q(r)}\left\|s_{i-1}\right\|_{r}^{2} .
\end{aligned}
$$

Thus

$$
\begin{aligned}
\left\|F\left(p_{i}\right)\right\|_{r} & \leq \frac{1}{2} \beta_{Q(r) r}^{n \times m}\left\|s_{i-1}\right\|_{r}^{2}\left\|\left(\mathrm{H}_{1}, \ldots, \mathrm{H}_{m}\right)^{t}\right\|_{r} \\
& =\frac{1}{2} \beta_{Q(r) r}^{n \times m}\left\|s_{i-1}\right\|_{r}^{2} \mathrm{H}^{r} .
\end{aligned}
$$

Then, by definition of $s_{i}$ and inequality (26), we get

$$
\begin{aligned}
\left\|s_{i}\right\|_{r} & =\left\|\operatorname{Jac}_{F}^{\dagger}\left(p_{i}\right) F\left(p_{i}\right)\right\|_{r} \\
& \leq\left\|\operatorname{Jac}_{F}^{\dagger}\left(p_{i}\right)\right\|_{r}\left\|F\left(p_{i}\right)\right\|_{r} \\
& \leq \mathrm{J}^{r}\left\|F\left(p_{i}\right)\right\|_{r} \\
& \leq \frac{1}{2} \beta_{Q(r) r}^{n \times m} \mathrm{~J}^{r} \mathrm{H}^{r}\left\|s_{i-1}\right\|_{r}^{2} .
\end{aligned}
$$


Now, we define $\tau_{i}:=\frac{\left\|s_{i}\right\|_{r}}{\left\|s_{i-1}\right\|_{r}}$, and we bound it

$$
\begin{aligned}
\tau_{i} & \leq \frac{1}{2} \beta_{Q(r) r}^{n \times m} \mathrm{~J}^{r} \mathrm{H}^{r}\left\|s_{i-1}\right\|_{r} \\
& \leq \frac{1}{2} \beta_{Q(r) r}^{n \times m} \mathrm{~J}^{r} \mathrm{H}^{r} \mathrm{~J}^{r}\left\|F\left(p_{i-1}\right)\right\|_{r} \\
& <\frac{1}{2} \beta_{Q(r) r}^{n \times m}\left(\mathrm{~J}^{r}\right)^{2} \mathrm{H}^{r}\|F(p)\|_{r},
\end{aligned}
$$

where the last inequality follows from the working assumption $\left\|F\left(p_{i-1}\right)\right\|_{r}<\|F(p)\|_{r}$. Since $\beta_{Q(r) r}^{m \times 1} \geq 1$ (see Corollary $2.8(1)$ ), we get

$$
\|F(p)\|_{r}<B_{2 r}=\frac{2}{\mathrm{~J}^{r}\left(\frac{2}{R}+\beta_{Q(r) r}^{m \times 1} \beta_{Q(r) r}^{n \times m} \mathrm{~J}^{r} \mathrm{H}^{r}\right)}<\frac{2}{\beta_{Q(r) r}^{n \times m}\left(\mathrm{~J}^{r}\right)^{2} \mathrm{H}^{r}} .
$$

Hence, 27] reads

$$
\tau_{i}<\frac{1}{2} \beta_{Q(r) r}^{n \times m}\left(\mathrm{~J}^{r}\right)^{2} \mathrm{H}^{r}\|F(p)\|_{r}<1 .
$$

Let $\tau:=\max _{i=1, \ldots, k}\left\{\tau_{i}\right\}$. We bound $\left\|\left(p_{k+1}-p\right)^{t}\right\|_{r}$ as follows:

$$
\begin{aligned}
\left\|\left(p_{k+1}-p\right)^{t}\right\|_{r} & \leq\left\|s_{0}\right\|_{r}+\left\|s_{1}\right\|_{r}+\cdots+\left\|s_{k}\right\|_{r} \\
& =\left\|s_{0}\right\|_{r}+\tau_{1}\left\|s_{0}\right\|_{r}+\tau_{1} \tau_{2}\left\|s_{0}\right\|_{r}+\cdots+\tau_{1} \tau_{2} \ldots \tau_{k}\left\|s_{0}\right\|_{r} \\
& =\left\|s_{0}\right\|_{r}\left(1+\tau_{1}+\tau_{1} \tau_{2}+\cdots+\tau_{1} \tau_{2} \ldots \tau_{k}\right) \\
& \leq\left\|s_{0}\right\|_{r} \sum_{i=0}^{k} \tau^{i}<\left\|s_{0}\right\|_{r} \sum_{i=0}^{\infty} \tau^{i}=\frac{\left\|s_{0}\right\|_{r}}{1-\tau} \leq \frac{\mathrm{J}^{r}\|F(p)\|_{r}}{1-\tau} .
\end{aligned}
$$

Then, by inequality (27), we find

$$
\begin{aligned}
\left\|\left(p_{k+1}-p\right)^{t}\right\|_{r} & <\frac{\mathrm{J}^{r}\|F(p)\|_{r}}{1-\frac{1}{2} \beta_{Q(r) r}^{n \times m}\left(\mathrm{~J}^{r}\right)^{2} \mathrm{H}^{r}\|F(p)\|_{r}} \\
& =\frac{2 \mathrm{~J}^{r}\|F(p)\|_{r}}{2-\beta_{Q(r) r}^{n \times m}\left(\mathrm{~J}^{r}\right)^{2} \mathrm{H}^{r}\|F(p)\|_{r}} .
\end{aligned}
$$

Again, by assumption, and using again that $\beta_{Q(r) r}^{m \times 1} \geq 1$,

$$
\|F(p)\|_{r}<B_{2 r} \leq \frac{2 R}{\mathrm{~J}^{r}\left(2+\beta_{Q(r) r}^{n \times m} R \mathrm{~J}^{r} \mathrm{H}^{r}\right)},
$$

so that inequality 30 reads $\left\|\left(p_{k+1}-p\right)^{t}\right\|_{r}<R$, showing that $p_{k+1} \in \mathcal{D}_{r}(p, R)$.

Next, we prove that $\left\|F\left(p_{k+1}\right)\right\|_{r}<\left\|F\left(p_{k}\right)\right\|_{r}$. Exactly the same argument as in Step I to show inequality (23), gives now

$$
\left\|F\left(p_{k+1}\right)\right\|_{r}<\frac{1}{2} \beta_{Q(r) r}^{m \times 1} \beta_{Q(r) r}^{n \times m} B_{2 r}\left(\mathrm{~J}^{r}\right)^{2} \mathbf{H}^{r}\left\|F\left(p_{k}\right)\right\|_{r} .
$$

Then, the result follows from (24).

Step III (Conclusion). If there exists $k \in \mathbb{N} \backslash\{0\}$ such that $\left\|F\left(p_{k}\right)\right\|_{r}=0$ the result holds, since then the zero locus $V(F)=\left\{\left(f_{1}=\cdots=f_{m}=0\right\}\right.$ contains the point $p_{k}$. 
We assume that $\left\|F\left(p_{k}\right)\right\|_{r} \neq 0$ for each $p_{k}$ 's. Hence we know from Step II that $\tau_{k}:=$

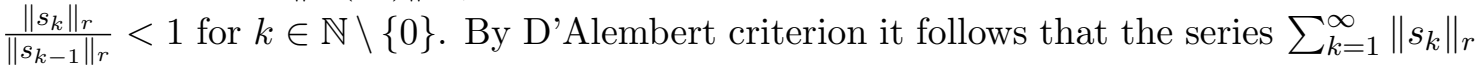
converges, so that $\lim _{k \rightarrow \infty}\left(\sum_{i=k+1}^{\infty}\left\|s_{i}\right\|_{r}\right)=0$. Define $p^{* t}:=p^{t}+\sum_{k=1}^{\infty} s_{k}$. Then, since $p_{k}^{t}=p^{t}+\sum_{i=1}^{k} s_{i}$, one has

$$
\lim _{k \rightarrow \infty}\left\|\left(p_{k}-p^{*}\right)^{t}\right\|_{r}=\lim _{k \rightarrow \infty}\left(\left\|\sum_{i=k+1}^{\infty} s_{i}\right\|_{r}\right) \leq \lim _{k \rightarrow \infty}\left(\sum_{i=k+1}^{\infty}\left\|s_{i}\right\|_{r}\right)=0 .
$$

Thus the sequence of points $\left\{p_{k}\right\}_{k \in \mathbb{N}}$ converges to the point $p^{*}=\lim _{k \rightarrow \infty} p_{k}$. Since the $p_{k}$ 's belong to $\mathcal{D}_{r}(p, R)$, and $R<\varepsilon$, the point $p^{*}$ belongs to the closure $\mathbf{B}_{r}(p, R) \subseteq \mathbf{C}_{r}(p, \varepsilon)$. We also know that $\left\|s_{k}\right\|_{r}=\tau_{1} \tau_{2} \ldots \tau_{k}\left\|s_{0}\right\|_{r} \leq \tau^{k}\left\|s_{0}\right\|_{r}$, where by (28) one has

$$
\tau:=\sup _{k \in \mathbb{N}}\left\{\tau_{k}\right\} \leq \frac{1}{2} \beta_{Q(r) r}^{n \times m}\left(\mathrm{~J}^{r}\right)^{2} \mathrm{H}^{r}\|F(p)\|_{r}<1 .
$$

Therefore $\lim _{k \rightarrow \infty}\left\|s_{k}\right\|_{r} \leq \lim _{k \rightarrow \infty} \tau^{k}\left\|s_{0}\right\|_{r}=0$. From inequality (26), we then conclude that

$$
\left\|F\left(p^{*}\right)\right\|_{r}=\left\|F\left(\lim _{k \rightarrow \infty} p_{k}\right)\right\|_{r}=\lim _{k \rightarrow \infty}\left\|F\left(p_{k}\right)\right\|_{r} \leq \frac{1}{2} \beta_{Q(r) r}^{n \times m} \mathrm{H}^{r} \lim _{k \rightarrow \infty}\left\|s_{k-1}\right\|_{r}^{2}=0 .
$$

Thus the zero locus $V(F)=\left\{f_{1}=\cdots=f_{m}=0\right\}$ crosses the cell $\mathbf{C}_{r}(p, \varepsilon)$. This completes the proof.

Q.E.D.

Remark 4.7 Combining Theorem 4.6 and $(6)$ we get

$$
B_{2 r}= \begin{cases}\frac{2 R}{J^{r}\left(2+m^{\frac{2}{r}-1} R \mathrm{~J}^{r} \mathrm{H}^{r}\right)} & \text { if } r \in[1,2), \\ \frac{2 R}{\mathrm{~J}^{r}\left(2+(m n)^{1-\frac{2}{r}} R \mathrm{~J}^{r} \mathrm{H}^{r}\right)} & \text { if } r \in[2, \infty] .\end{cases}
$$

Similarly, as we did for the computation of $\mathrm{H}^{r}$ in the previous section, we will estimate randomly the value of $\mathrm{J}^{r}$.

Example 4.8 We consider the quadric defined by the polynomial $F=f(x, y, z)=x^{2}+y+$ $z \in \mathbb{R}[x, y, z]$, and the point $p=(0.647,0.647,-1.254) \in \mathbb{R}^{3}$. We observe that $\operatorname{deg}(F)=2$, so the Hessian $H_{F}(\boldsymbol{x})$ is not the zero matrix, and that $\operatorname{Jac}_{F}(p)=(1.29,1,1)$ has full rank. Therefore the hypotheses of Theorem 4.6 are satisfied. Let $r \in\{1,2, \infty\}$. We start with $\varepsilon=0.5$ and we iteratively reduce it; we stop when the inequality of Theorem 4.6 is not any more satisfied. The results appear in Table 4 . The table shows that

$$
\mathbf{B}_{1}(p, 0.25) \cap V(F) \neq \emptyset, \quad \mathbf{B}_{2}(p, 0.2) \cap V(F) \neq \emptyset, \quad \mathbf{B}_{\infty}(p, 0.1) \cap V(F) \neq \emptyset .
$$

A notable special case is that of linear varieties. 


\begin{tabular}{c|c|c|c}
\hline$r$ & $\varepsilon$ & $B_{2 r}$ & $\|F(p)\|_{r}$ \\
\hline \hline 1 & 0.25 & 0.191 & 0.188 \\
\hline 2 & 0.2 & 0.299 & 0.188 \\
\hline$\infty$ & 0.1 & 0.256 & 0.188 \\
\hline
\end{tabular}

Table 4: Results of the executions corresponding to Example 4.8

Remark 4.9 (The algebraic linear case) Notation as above. Here, we analyze the case when all the functions $f_{j}=f_{j}(\boldsymbol{x})$ are linear polynomials. We see that the bound $B_{1 r}$ of Theorem 3.2 simply becomes $B_{1 r}=\left\|\operatorname{Jac}_{F}(p)\right\|_{r} \varepsilon$.

Concerning sufficient crossing cell conditions, we observe that $\operatorname{Jac}_{F}(\boldsymbol{x})$ has full rank $m$ for each $\boldsymbol{x}$ under the only hypothesis that $\operatorname{rank}\left(\operatorname{Jac}_{F}(p)\right)=m$ (compare with Lemma 4.5). Similarly, Theorem 4.6 holds true under the only assumption that $R<\varepsilon$ and does not require the assumption that the Hessian matrix $H_{F}(\boldsymbol{x})$ is not the zero matrix. Moreover, the bound $B_{2 r}$ simply becomes $B_{2 r}=\frac{R}{\mathrm{~J} r}$. Indeed, for linear polynomials $f_{j}$, the same argument as in Step I of the theorem shows that the linear variety $V(F)$ crosses the cell $\mathbf{C}(p, \varepsilon)$ as soon as $\|F(p)\|_{r}<\frac{R}{\mathrm{~J}^{r}}$ (since inequality $(22)$ yields $\left\|F\left(p_{1}\right)\right\|_{r}=0$, whence $f_{j}\left(p_{1}\right)=0$ for each $j=1, \ldots, m$, in that case).

Example 4.10 (Spatial line) In the affine space $\mathbb{A}_{(x, y, z)}^{3}(\mathbb{R})$, consider the line $\ell$ of equations $x-z-2=y-3 z+1=0$. Letting $F=(x-z-2, y-3 z+1)^{t}$, compute

$$
\operatorname{Jac}_{F}(x, y, z)=\left(\begin{array}{ccc}
1 & 0 & -1 \\
0 & 1 & -3
\end{array}\right)
$$

and

$$
\begin{aligned}
\operatorname{Jac}_{F}^{\dagger}(x, y, z) & =\left(\begin{array}{cc}
1 & 0 \\
0 & 1 \\
-1 & -3
\end{array}\right)\left[\left(\begin{array}{ccc}
1 & 0 & -1 \\
0 & 1 & -3
\end{array}\right)\left(\begin{array}{cc}
1 & 0 \\
0 & 1 \\
-1 & -3
\end{array}\right)\right]^{-1} \\
& =\left(\begin{array}{cc}
1 & 0 \\
0 & 1 \\
-1 & -3
\end{array}\right)\left(\begin{array}{cc}
2 & 3 \\
3 & 10
\end{array}\right) \\
& =\left(\begin{array}{cc}
1 & 0 \\
0 & 1 \\
-1 & -3
\end{array}\right) \frac{1}{11}\left(\begin{array}{cc}
10 & -3 \\
-3 & 2
\end{array}\right)=\frac{1}{11}\left(\begin{array}{cc}
10 & -3 \\
-3 & 2 \\
-1 & -3
\end{array}\right) .
\end{aligned}
$$

Then

$$
\left\|\operatorname{Jac}_{F}(x, y, z)\right\|_{\infty}=4 \quad \text { and } \quad\left\|\operatorname{Jac}_{F}^{\dagger}(x, y, z)\right\|_{\infty}=\frac{13}{11}
$$

Now, fix $\varepsilon=\frac{2}{5}$. The quantities $B_{1 \infty}, B_{2 \infty}$, introduced in Theorems 3.2 and 4.6 (see also Remarks 3.3 and 4.7 , are $B_{1 \infty}=4 \varepsilon=1.6$ and $B_{2 \infty}=\frac{11}{13} \varepsilon \approx 0.3385$, respectively.

If we take $p=(0,0,0)$, then $\|F(p)\|_{\infty}=\left\|(-2,1)^{t}\right\|_{\infty}=2$, so that $\|F(p)\|_{\infty}>B_{1 \infty}$, whence $\ell$ does not cross the cell $\mathbf{C}\left(p, \frac{2}{5}\right)$ (see Figure 1, panel (a)).

If we take $p=(2.1,-1,0.1)$, then $\|F(p)\|_{\infty}=\left\|\left(0,-\frac{3}{10}\right)^{t}\right\|_{\infty}=\frac{3}{10}$, so that $\|F(p)\|_{\infty}<$ $B_{2 \infty}$, whence $\ell$ crosses the cell $\mathbf{C}\left(p, \frac{2}{5}\right)$ (see Figure 1 , panel (a)). 
Indeed, note that in this case the $\infty$-distance $d_{\infty}(\ell, p)$ of $\ell$ from the point $p=$ $(2.1,-1,0.1)$ is $\frac{3}{40}=0.075$. We compute $d_{\infty}(\ell, p)$ by using the parametrization $(t+2,3 t-1, t)$ of the spatial line $\ell$, which gives

$$
\begin{aligned}
d_{\infty}(\ell, p) & =\min _{q \in \ell}\left\|(q-p)^{t}\right\|_{\infty}=\min _{t \in \mathbb{R}}\left\|\left(\begin{array}{c}
t+2 \\
3 t-1 \\
t
\end{array}\right)-\left(\begin{array}{c}
2.1 \\
-1 \\
0.1
\end{array}\right)\right\|_{\infty}=\min _{t \in \mathbb{R}}\left\|\left(\begin{array}{c}
t-0.1 \\
3 t \\
t-0.1
\end{array}\right)\right\|_{\infty} \\
& =\min _{t \in \mathbb{R}}\{\max \{|t-0.1|, 3|t|\}\}=\min _{t \in \mathbb{R}}\{g(t)\} .
\end{aligned}
$$

The plot of the curve $y=g(t)$ is shown in Figure 1, panel (b); its minimum is attained at $t=\frac{1}{40}$, so that it is equal to $\frac{3}{40}$.

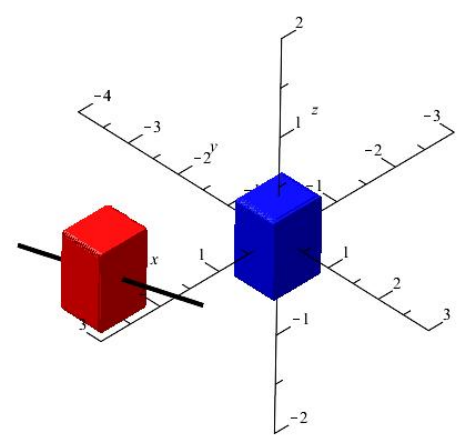

(a)

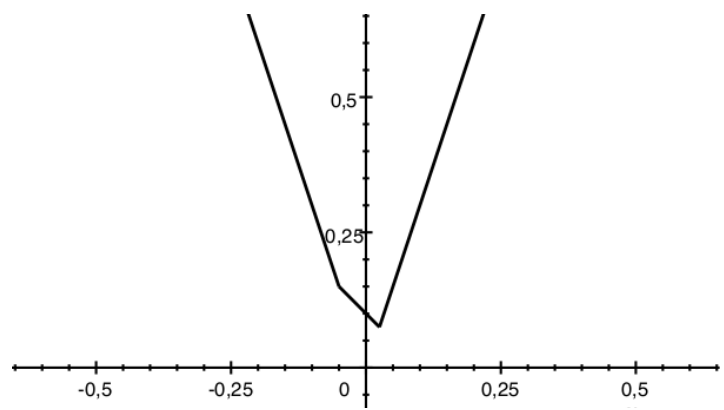

(b)

Figure 1: Plot of $\ell$ and $\infty$-balls centered at $(0,0,0),(2.1,-1,0.1)$ and of radius $\frac{2}{5}$, panel (a), and plot of $y=g(t)=\max \{|t-0.1|, 3|t|\}$, panel (b).

In the case of algebraic hypersurfaces and $\infty$-norm, let us compare with the results of [16. Proposition 3.2], [17.

Remark 4.11 In the special case of algebraic hypersurfaces and $\infty$-norm, the quantity $B_{2}$ was taken as $B_{2}=\frac{2 R}{\mathrm{~J}\left(c+n^{5 / 2} \mathrm{HJR}\right)}$, where $c=\max \{2, \sqrt{n}\}$ (see [17]). Such a quantity is smaller than the corresponding quantity $B_{2 \infty}=\frac{2 R}{\mathrm{~J}^{\infty}\left(2+n R \mathrm{~J}^{\infty} \mathrm{H}^{\infty}\right)}$ as in Remark 4.7 with $m$ fixed to 1 (so that the assumption in [16, Proposition 3.2], [17] is stronger). The reason is that the proof presented here in the general case differs from that of [16, Proposition 3.2] leading to a slight improvement in the special case taken into account.

\section{$5 \quad r$-norm distances}

Notations and assumptions as in the previous sections. In particular, let $F=\left(f_{1}, \ldots, f_{m}\right)^{t}$, where $f_{1}, \ldots, f_{m} \in \mathcal{A}(U)$ are real analtytic functions on a convex open set $U \subseteq \mathbb{R}^{n}$, with $m \leq n$. We assume that the $f_{j}$ ' are not all linear polynomials (referring to Remark 4.9 for this special case), and, as in Section 4 , that $\operatorname{Jac}_{F}(p)$ has full rank $m$ and that the Hessian $H_{F}(\boldsymbol{x})$ is not the zero matrix. 
Let's start comparing the bounds $B_{1 r}$ and $B_{2 r}$ introduced in theorems 3.2 and 4.6 , respectively. For this purpose, in the sequel, we will assume that $R$ is a positive real number satisfying the inequality appearing in Theorem 4.6.

Proposition 5.1 Let $U \subseteq \mathbb{R}^{n}$ be a convex open set, let $f_{j}=f_{j}(\boldsymbol{x}) \in \mathcal{A}(U), j=1, \ldots, m$, $m \leq n$, and let $F=\left(f_{1}, \ldots, f_{m}\right)^{t}$. Assume that the Hessian $H_{F}(\boldsymbol{x})$ is not the zero matrix. Let $p$ be a point of $U$, and let $\mathbf{C}_{r}(p, \varepsilon) \subseteq \mathbf{B}_{r}(p, \varepsilon)$ be an $(r, \boldsymbol{\varepsilon})$-cell centered at $p$. Suppose that $F$ and $p$ satisfy the hypotheses of theorems 3.2 and 4.6. Then the bounds $B_{1 r}$ and $B_{2 r}$ satisfy the inequality

$$
B_{2 r}<B_{1 r}
$$

Proof. To see this, note that

$$
B_{2 r}<\frac{2 R}{2 \mathrm{~J}^{r}}=\frac{R}{\max _{\boldsymbol{x} \in \mathbf{B}_{r}(p, R)}\left\{\left\|\operatorname{Jac}_{F}^{\dagger}(\boldsymbol{x})\right\|_{r}\right\}} \leq \frac{R}{\left\|\operatorname{Jac}_{F}^{\dagger}(p)\right\|_{r}}<\frac{\varepsilon}{\left\|\operatorname{Jac}_{F}^{\dagger}(p)\right\|_{r}} .
$$

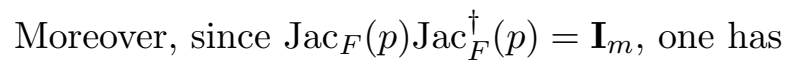

$$
1=\left\|\mathbf{I}_{m}\right\|_{r}=\left\|\operatorname{Jac}_{F}(p) \operatorname{Jac}_{F}^{\dagger}(p)\right\|_{r} \leq\left\|\operatorname{Jac}_{F}(p)\right\|_{r}\left\|\operatorname{Jac}_{F}^{\dagger}(p)\right\|_{r}
$$

whence

$$
\frac{1}{\left\|\operatorname{Jac}_{F}^{\dagger}(p)\right\|_{r}} \leq\left\|\operatorname{Jac}_{F}(p)\right\|_{r}
$$

By combining with inequality (32), we then find $B_{2 r}<\left\|\operatorname{Jac}_{F}(p)\right\|_{r} \varepsilon<B_{1 r}$.

Q.E.D.

We aim to use the bounds $B_{1 r}$ and $B_{2 r}$ to decide whether or not the zero locus $V(F)=$ $\left\{f_{1}=\cdots=f_{m}=0\right\}$ intersects a given $(r, \varepsilon)$-cell $\mathbf{C}_{r}(p, \varepsilon) \subseteq \mathbf{B}_{r}(p, \varepsilon)$. From Proposition 5.1 the following three cases can occur: either $\|F(p)\|_{r}<B_{2 r}$ (crossing case), or $B_{2 r} \leq$ $\|F(p)\|_{r} \leq B_{1 r}$ (uncertain case), or $\|F(p)\|_{r}>B_{1 r}$ (not crossing case). This leads to three possible outputs of the algorithm, namely:

- 0 if the zero locus $V(F)$ does not cross $\mathbf{C}(p, \varepsilon)$.

- 1 if the zero locus $V(F)$ crosses $\mathbf{C}(p, \varepsilon)$.

- $\zeta$ (unknown) if neither Theorem 3.2 nor Theorem 4.6 applies.

The following algorithm summarizes the above ideas. For this purpose, we observe that the bounds $B_{1 r}$ and $B_{2 r}$ depend on $F, p$ and $\varepsilon$. To emphasize this fact, we will write $B_{j r}(F, p, \varepsilon)$ for $j=1,2$. 


\section{The Crossing Cell Algorithm}

Given an open convex set $U \subseteq \mathbb{R}^{n}, F=\left(f_{1}, \ldots, f_{m}\right)^{t}$ made up of $m \leq n$ real analytic functions $f_{j}$ on $U$ such that the Hessian $H_{F}(\boldsymbol{x})$ is not the zero matrix, a point $p \in U$ such that the $\operatorname{Jacobian} \operatorname{Jac}_{F}(p)$ has full rank $m$, a tolerance value $\varepsilon>0$, and $r \in[1, \infty]$, the algorithm returns an element of $\{0,1, \zeta\}$.

1. Compute $\|F(p)\|_{r}$, and the bounds $B_{1 r}(F, p, \varepsilon)$ and $B_{2 r}(F, p, \varepsilon)$ in Theorems 3.2 and 4.6 (see also Remark 4.9).

2. If $\|F(p)\|_{r}>B_{1 r}(F, p, \varepsilon)$ then OUT $=0$;

if $\|F(p)\|_{r}<B_{2 r}(F, p, \varepsilon)$ then OUT $=1$;

else OUT $=\zeta$.

3. Return OUT.

To overcome the uncertain situation when the Crossing Cell Algorithm's output is $\zeta$, we suggest a heuristic procedure, based on the following steps.

1. Assumption. Let $B_{2 r}(F, p, \varepsilon) \leq\|F(p)\|_{r} \leq B_{1 r}(F, p, \varepsilon)$, so that neither Theorem 3.2 nor Theorem 4.6 applies to conclude that $V(F)$ crosses/does not cross the considered $(r, \varepsilon)$-cell $\mathbf{C}_{r}(p, \varepsilon)$ centered at $p$.

2. Passing to the $\infty$-norm. We consider the $(\infty, \varepsilon)$-ball $\mathbf{B}_{\infty}(p, \varepsilon)$ centered at $p$ (see Definition 3.1) which clearly contains the given $(r, \varepsilon)$-cell $\mathbf{C}_{r}(p, \varepsilon)$ (the fact that, for any $r \geq 1, \mathbf{B}_{r}(p, \varepsilon) \subset \mathbf{B}_{\infty}(p, \varepsilon)$ is a consequence of Proposition 2.3 (1), first inequality).

3. Subdivision of the $(\infty, \varepsilon)$-ball. We consider the $\left(\infty, \frac{\varepsilon}{2}\right)$-balls $\mathbf{B}_{\infty}\left(q_{i}, \frac{\varepsilon}{2}\right) \subset \mathbf{B}_{\infty}(p, \varepsilon)$ centered at the points $q_{i}, i=1, \ldots, 2^{n}$, defined as follows. Let $p=\left(p_{1}, \ldots, p_{n}\right)$, let $S=\{0,1\}^{n}$ be a set of (ordered) $n$-tuples consisting of 0 's and 1's and let $s_{i}=$ $\left(s_{i 1}, \ldots, s_{i n}\right)$ be the $i$-th element of $S$. For each $i=1, \ldots, 2^{n}$, set $q_{i}:=\left(q_{i 1}, \ldots, q_{i n}\right)$, where (see Figure 2, panels (a), (b))

$$
q_{i k}=p_{k}+(-1)^{s_{i k}} \frac{\varepsilon}{2}, \quad k=1, \ldots, n .
$$

Note that the use of the $\infty$-norm allows us to conclude that

$$
\bigcup_{i=1}^{2^{n}} \mathbf{B}_{\infty}\left(q_{i}, \frac{\varepsilon}{2}\right)=\mathbf{B}_{\infty}(p, \varepsilon) \text {. }
$$

4. Crossing check. We now address the crossing problem of the zero locus $V(F)$ on the (smaller) $\infty$-balls $\mathbf{B}_{\infty}\left(q_{i}, \frac{\varepsilon}{2}\right), i \in\left\{1, \ldots, 2^{n}\right\}$. The following three possibilities can occur.

4.1 We have $\left\|F\left(q_{i}\right)\right\|_{\infty}<B_{2 \infty}\left(F, q_{i}, \frac{\varepsilon}{2}\right)$ for at least one index $i$ : we conclude that $V(F)$ crosses $\mathbf{B}_{\infty}(p, \varepsilon)$.

4.2 We have $\left\|F\left(q_{i}\right)\right\|_{\infty}>B_{1 \infty}\left(F, q_{i}, \frac{\varepsilon}{2}\right)$ for all indices $i$ : we conclude that $V(F)$ does not cross $\mathbf{B}_{\infty}(p, \varepsilon)$. 


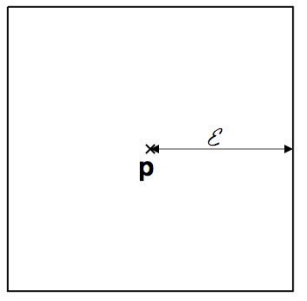

(a)

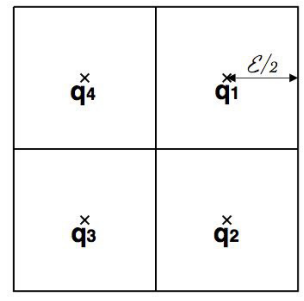

(b)

\begin{tabular}{|c|c|c|c|}
\hline$q^{\times} 10$ & $\mathbf{q}_{111}^{\times}$ & $\mathrm{q}_{12}^{\mathrm{x}}$ & $\underset{q}{q} / 4$ \\
\hline$q_{9}^{x}$ & $q_{11}^{x}$ & $q_{13}^{x}$ & $q_{2}^{x}$ \\
\hline$q_{8}^{x}$ & $q^{x} 15$ & $q_{14}^{\times}$ & $\mathbf{q}^{x}$ \\
\hline $\mathbf{q}_{7}^{x}$ & $q^{x}$ & $q_{5}^{x}$ & $q_{4}^{x}$ \\
\hline
\end{tabular}

(c)

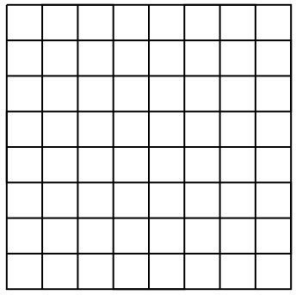

(d)

Figure 2: Example of the subdivision procedure: an $(\infty, \varepsilon)$-ball centered at $p$, panel (a), and the representation of the first three subdivision steps, panels (b), (c) and (d).

4.3 None of the above two cases occurs: we go back to the subdivision procedure as in step 3), we subdivide each $\mathbf{B}_{\infty}\left(q_{i}, \frac{\varepsilon}{2}\right)$ into $2^{n}$ smaller $\infty$-balls (see Figure 2 , panel (c)) and we apply the Crossing Cell Algorithm to such smaller $\infty$-balls.

We gather the described procedure in the following algorithm.

\section{The Iterative Crossing Cell Algorithm}

Given an open convex set $U \subseteq \mathbb{R}^{n}, F=\left(f_{1}, \ldots, f_{m}\right)^{t}$ made up of $m \leq n$ real analytic functions $f_{j}$ over $U$ such that the Hessian $H_{F}(\boldsymbol{x})$ is not the zero matrix, a point $p=$ $\left(p_{1}, \ldots, p_{n}\right) \in U$ such that the $\operatorname{Jacobian} \operatorname{Jac}_{F}(p)$ has full rank $m$, tolerance values $\varepsilon>$ $2 \delta>0$, and $r \in[1, \infty]$, the algorithm returns an element of $\{0,1, \zeta\}$.

1. Apply the Crossing Cell Algorithm to $(F, p, \varepsilon, r)$ and let OUT be its output.

2. If OUT $\in\{0,1\}$ then return OUT and stop;

else consider the $(\infty, \varepsilon)$-ball $\mathbf{B}_{\infty}(p, \varepsilon)$, set $\varepsilon^{\prime}=\varepsilon$, let $S=\{0,1\}^{n}$, and denote by $s_{i}=\left(s_{i 1}, \ldots, s_{i n}\right)$ its $i$-th element.

3. For each $i=1, \ldots, 2^{n}$, consider the $\left(\infty, \frac{\varepsilon^{\prime}}{2}\right)$-balls $\mathbf{B}_{\infty}\left(q_{i}, \frac{\varepsilon^{\prime}}{2}\right)$ centered at $q_{i}=$ $\left(q_{i 1}, \ldots, q_{i n}\right)$, where

$$
q_{i k}=p_{k}+(-1)^{s_{i k}} \frac{\varepsilon^{\prime}}{2}, \quad k=1, \ldots, n
$$

4. Apply the Crossing Cell Algorithm to $\left(F, q_{i}, \frac{\varepsilon^{\prime}}{2}, \infty\right)$ for each index $i$, and let OUTVector be the vector of the outputs.

5. If OUTVector has at least one entry equal to 1 , then return OUT $=1$ and stop.

If OUTVector has all entries equal to 0 , then return OUT $=0$ and stop.

Else $\varepsilon^{\prime} \leftarrow \frac{\varepsilon^{\prime}}{2}$.

If $\varepsilon^{\prime}>\delta$ then for each index $i$ set $p=q_{i}$, and go to step 3;

else return OUT $=\zeta$;

Here is a first simple example. The tables below sum up the values of meaningful variables for each iteration: $R$ denotes the value of the radius as defined in Lemma 4.5, 
$B_{1 \infty}$ and $B_{2 \infty}$ are the bounds provided in theorems 3.2 and 4.6 , and OUT is the algorithm's output.

Example 5.2 We consider the hypersurface defined by the polynomial $F=f(x, y, z)=$ $x^{3}+y+z \in \mathbb{R}[x, y, z]$, and the point $p=(1,1,-0.9) \in \mathbb{R}^{3}$. We let $\varepsilon=1.07$ and $r=\infty$. We observe that $\operatorname{deg}(f)=3$, so the Hessian $H_{F}(\boldsymbol{x})$ is not the zero matrix, and that $\operatorname{Jac}_{F}(p)=(3,1,1)$ has full rank; therefore the hypotheses of theorems 3.2 and 4.6 are satisfied. We start applying the Crossing Cell Algorithm to $(F, p, \varepsilon, \infty)$ which returns the value $\zeta$. Indeed, a direct computation shows that we are in the uncertain case $B_{2 \infty} \leq\|F(p)\|_{\infty} \leq B_{1 \infty}$ (see Table 5).

\begin{tabular}{c|c|c|c|c|c|c}
\hline$p$ & $\|F(p)\|_{\infty}$ & $\varepsilon$ & $B_{1 \infty}$ & $R$ & $B_{2 \infty}$ & OUT \\
\hline$(1,1,-0.9)$ & 1.100 & 1.07 & 26.569 & 0.240 & 0.077 & $\zeta$ \\
\hline
\end{tabular}

Table 5: Application of the Crossing Cell Algorithm to $\left(F=x^{3}+y+z, p=(1,1,-0.9), \varepsilon, \infty\right)$.

Now, take $\delta=0.1$. We apply the Iterative Crossing Cell Algorithm to $(F, p, \varepsilon, \delta, \infty)$ whose direct computations are gathered in Table 6. In the first iteration, the Crossing Cell Algorithm is applied to $F$ on the $2^{3}=8 \infty$-balls centered at the points $q_{i}$ with radius $\varepsilon^{\prime}=\varepsilon / 2=0.535$. The OUTVector only contains values $\zeta$, meaning that in each ball the algorithm gives an undecidable result. In the second iteration, the Crossing Cell Algorithm is applied to $F$ on the $2^{6}=64 \infty$-balls centered at the new points $q_{i}$ with radius $\varepsilon^{\prime}=\varepsilon / 4=0.2675$. Table 5 shows the computations until we find the first 1 entry of OUTVector. These computations show that the crossing problem is undecidable for $B_{\infty}((0.198,0.198,-0.633), 0.2675)$, while

$$
\begin{aligned}
& \mathbf{B}_{\infty}((0.198,0.198,-1.703), 0.2675) \cap V(F)=\emptyset \\
& \mathbf{B}_{\infty}((0.198,0.198,-1.168), 0.2675) \cap V(F)=\emptyset \\
& \mathbf{B}_{\infty}((0.198,0.198,-0.098), 0.2675) \cap V(F) \neq \emptyset .
\end{aligned}
$$

We then conclude that the hypersurface of equation $x^{3}+y+z=0$ crosses the $\infty$-ball $\mathbf{B}_{\infty}(p, \varepsilon)$.

To conclude this section, we illustrate the Iterative Crossing Cell Algorithm by means of the examples introduced in Section 3 .

Example 5.3 We consider the zero-dimensional set introduced in Example 3.5. So, $f_{1}=$ $x^{2}+y^{2}-1, f_{2}=y-2 x^{2}$ and $F=\left(f_{1}, f_{2}\right)^{t}$. As we have seen the real variety $V(F)$ consists in two points, that can be approximated as $V(F) \approx\{(0.625,0.780),(-0.625,0.780)\}$.

We consider the point $p=(0.5,0.7), \varepsilon=0.5$, and $r=\infty$. We observe that $\operatorname{deg}\left(f_{1}\right)=$ $\operatorname{deg}\left(f_{2}\right)=2$, so the Hessian $H_{F}(\boldsymbol{x})$ is not the zero matrix, and that $\operatorname{Jac}_{F}(p)$ has full rank; therefore the hypotheses of theorems 3.2 and 4.6 are satisfied. We start applying the Crossing Cell Algorithm to $(F, p, \varepsilon, \infty)$ which returns the value $\zeta$. Indeed, a direct computation shows that we are in the uncertain case $B_{2 \infty} \leq\|F(p)\|_{\infty} \leq B_{1 \infty}$ (see Table7).

Now, take $\delta=0.01$. We apply the Iterative Crossing Cell Algorithm to $(F, p, \varepsilon, \delta, \infty)$ which, in the first iteration, shows that the variety intersects one of the cells, while in the other the undecided situation continues. Direct computations are gathered in Table 8. 


\begin{tabular}{c|c|c|c|c|c|c|c}
\hline \multicolumn{7}{c}{ Iteration 1} \\
\hline$q_{i}$ & $\left\|F\left(q_{i}\right)\right\|_{\infty}$ & $\varepsilon^{\prime}$ & $B_{1 \infty}$ & $R$ & $B_{2 \infty}$ & OUTVector & OUT \\
\hline$(0.465,0.465,-1.435)$ & 0.869 & 0.535 & 3.985 & 0.166 & 0.073 & $\zeta$ & \\
$(0.465,0.465,-0.365)$ & 0.201 & 0.535 & 3.979 & 0.166 & 0.073 & $\zeta$ & \\
$(0.465,1.535,-1.435)$ & 0.201 & 0.535 & 3.979 & 0.166 & 0.073 & $\zeta$ & \\
$(0.465,1.535,-0.365)$ & 1.271 & 0.535 & 3.983 & 0.166 & 0.073 & $\zeta$ & \\
$(1.535,0.465,-1.435)$ & 2.647 & 0.535 & 10.172 & 0.530 & 0.133 & $\zeta$ & \\
$(1.535,0.465,-0.365)$ & 3.717 & 0.535 & 10.172 & 0.530 & 0.132 & $\zeta$ & \\
$(1.535,1.535,-1.435)$ & 3.717 & 0.535 & 10.181 & 0.530 & 0.132 & $\zeta$ & \\
$(1.535,1.535,-0.365)$ & 4.787 & 0.535 & 10.179 & 0.530 & 0.132 & $\zeta$ & \\
\hline \hline
\end{tabular}

\begin{tabular}{c|c|c|c|c|c|c|c}
\hline \hline \multicolumn{9}{c}{ Iteration 2} & & \\
\hline$q_{i}$ & $\left\|F\left(q_{i}\right)\right\|_{\infty}$ & $\varepsilon^{\prime}$ & $B_{1 \infty}$ & $R$ & $B_{2 \infty}$ & OUTVector & OUT \\
\hline$(0.198,0.198,-1.703)$ & 1.497 & 0.2675 & 0.865 & 0.265 & 0.341 & 0 & \\
$(0.198,0.198,-1.168)$ & 0.962 & 0.2675 & 0.864 & 0.265 & 0.341 & 0 & \\
$(0.198,0.198,-0.633)$ & 0.427 & 0.2675 & 0.864 & 0.265 & 0.137 & $\zeta$ & \\
$(0.198,0.198,-0.098)$ & 0.108 & 0.2675 & 0.865 & 0.265 & 0.137 & 1 & 1 \\
\hline
\end{tabular}

Table 6: Application of the Iterative Crossing Cell Algorithm to $\left(F=x^{3}+y+z, p=\right.$ $(1,1,-0.9), \varepsilon, \delta, \infty)$.

\begin{tabular}{c|c|c|c|c|c|c}
\hline$p$ & $\|F(p)\|_{\infty}$ & $\varepsilon$ & $B_{1 \infty}$ & $R$ & $B_{2 \infty}$ & OUT \\
\hline$(0.5,0.7)$ & 0.26 & 0.5 & 2.5 & 0.21 & 0.0631 & $\zeta$ \\
\hline
\end{tabular}

Table 7: Application of the Crossing Cell Algorithm to $(F, p, \varepsilon, \infty)$ in Example 5.3 .

Example 5.4 We consider the Viviani curve introduced in Example 3.6. So $f_{1}=x^{2}+y^{2}+$ $z^{2}-4, f_{2}=(x-1)^{2}+y^{2}-1$ and $F=\left(f_{1}, f_{2}\right)^{t}$. We consider the point $p=(2.1,-0.1,0.1)$, $\varepsilon=0.5$, and $r=\infty$. We observe that $\operatorname{deg}\left(f_{1}\right)=\operatorname{deg}\left(f_{2}\right)=2$, so the Hessian $H_{F}(\boldsymbol{x})$ is not the zero matrix, and that $\operatorname{Jac}_{F}(p)$ has full rank; therefore the hypotheses of theorems 3.2 and 4.6 are satisfied. We start applying the Crossing Cell Algorithm to $(F, p, \varepsilon, \infty)$ which returns the value $\zeta$. Indeed, a direct computation shows that we are in the uncertain case $B_{2 \infty} \leq\|F(p)\|_{\infty} \leq B_{1 \infty}$ (see Table 9).

Now, take $\delta=0.01$. We apply the Iterative Crossing Cell Algorithm to $(F, p, \varepsilon, \delta, \infty)$ which, in the first iteration, shows that the variety intersects one of the cells, while in the

\begin{tabular}{c|c|c|c|c|c|c|c}
\hline \multicolumn{8}{c}{ Iteration 1} \\
\hline$q_{i}$ & $\left\|F\left(q_{i}\right)\right\|_{\infty}$ & $\varepsilon^{\prime}$ & $B_{1 \infty}$ & $R$ & $B_{2 \infty}$ & OUTVector & OUT \\
\hline$(0.375,0.575)$ & 0.529 & 0.25 & 0.875 & 0.172 & 0.0312 & $\zeta$ & \\
\hline$(0.375,0.825)$ & 0.544 & 0.25 & 0.875 & 0.207 & 0.0262 & $\zeta$ & \\
\hline$(0.625,0.575)$ & 0.279 & 0.25 & 1.12 & 0.182 & 0.0578 & $\zeta$ & \\
\hline$(0.625,0.825)$ & 0.0712 & 0.25 & 1.12 & 0.239 & 0.106 & 1 & 1 \\
\hline
\end{tabular}

Table 8: First iteration of the Iterative Crossing Cell Algorithm when applied to $(F, p, \varepsilon, \infty)$ in Example 5.3 . 


\begin{tabular}{c|c|c|c|c|c|c}
\hline$p$ & $\|F(p)\|_{\infty}$ & $B_{1 \infty}$ & $R$ & $B_{2 \infty}$ & $\varepsilon$ & OUT \\
\hline$(2.1,-0.1,0.1)$ & 0.43 & 3.05 & 0.025 & 0.001 & 0.5 & $\zeta$ \\
\hline
\end{tabular}

Table 9: Application of the Crossing Cell Algorithm to $(F, p, \varepsilon, \infty)$ in Example 5.4 .

other the undecided situation continues. For the reader's convenience we summarize the output in Table 10.

\begin{tabular}{c|c|c|c|c|c|c|c|}
\hline \multicolumn{1}{c}{ Iteration 1} \\
\hline$q_{i}$ & $\left\|F\left(q_{i}\right)\right\|_{\infty}$ & $B_{1 \infty}$ & $R$ & $B_{2 \infty}$ & $\varepsilon^{\prime}$ & OUTVector & OUT \\
\hline$(1.98,-0.225,-0.025)$ & 0.0481 & 1.30 & 0.042 & 0.00205 & 0.25 & $\zeta$ & \\
\hline$(1.98,-0.225,0.225)$ & 0.00188 & 1.40 & 0.058 & 0.00531 & 0.25 & 1 & 1 \\
\hline$(1.98,0.025,-0.025)$ & 0.0981 & 1.20 & 0.006 & 0.0000655 & 0.25 & $\zeta$ & \\
\hline$(1.98,0.025,0.225)$ & 0.0488 & 1.30 & 0.041 & 0.00162 & 0.25 & $\zeta$ & \\
\hline$(2.22,-0.225,-0.025)$ & 1.00 & 1.42 & 0.036 & 0.00134 & 0.25 & $\zeta$ & \\
\hline$(2.22,-0.225,0.225)$ & 1.05 & 1.52 & 0.057 & 0.00415 & 0.25 & $\zeta$ & \\
\hline$(2.22,0.025,-0.025)$ & 0.952 & 1.32 & 0.006 & 0.0000583 & 0.25 & $\zeta$ & \\
\hline$(2.22,0.025,0.225)$ & 1.00 & 1.42 & 0.044 & 0.00188 & 0.25 & $\zeta$ & \\
\hline
\end{tabular}

Table 10: First iteration of the Iterative Crossing Cell Algorithm when applied to $(F, p, \varepsilon, \infty)$ in Example 5.4 .

Figure 3 gives a geometry's picture of the situation in Example 5.4 .

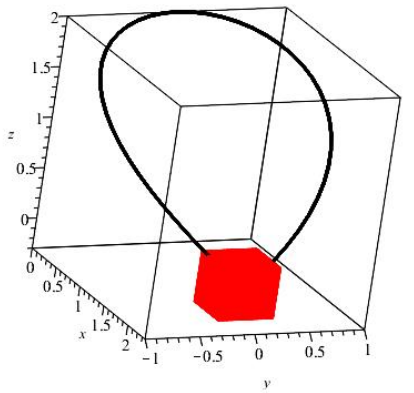

(a)

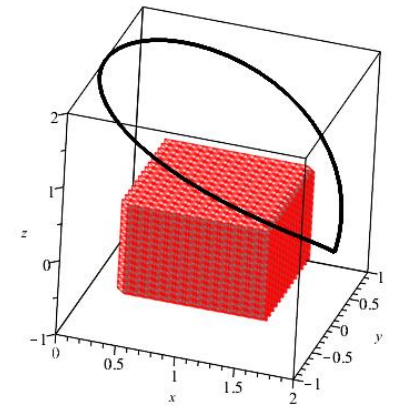

(b)

Figure 3: Viviani curve and $\mathbf{B}_{\infty}((1.98,-0.225,0.225), 0.25)$ as in Example 3.6 panel (a); Viviani curve and $\mathbf{B}_{\infty}((1,0,0), 0.63)$ as in Example 5.4 , panel (b).

Acknowledgements. A major part of this work was developed while J.R. Sendra was visiting, in the frame of GNSAGA-Istituto Nazionale di Alta Matematica, the University of Genova, and while M.C. Beltrametti and M. Torrente were visiting the University of Alcalá, in the frame of the project Giner de los Rios and of GNSAGA-Istituto Nazionale di Alta Matematica. J. R. Sendra is supported by the Spanish Ministerio de Economa y 
Competitividad, and by the European Regional Development Fund (ERDF), under the Project MTM201454141-P, and by the Univesity of Alcalá.

\section{References}

[1] T. Apostol, Mathematical Analysis, Second Edition, Addison-Wesley World Student Edition, Reading MA., 1974.

[2] M.C. Beltrametti, M. Piana, and A.M. Massone, Hough transform of special classes of curves, SIAM J. Imaging Sci. 6, (2013), 391-412.

[3] J.F. Canny, The complexity of robot motion planning, The MIT Press, 1988.

[4] H. Cartan, Elementary Theory of Analytic Functions of One or Several Complex Variables, Dover, 1995, unabridged and unaltered republication of the 1973 second printing of the work first published by the Addison-Wesley Publishing Company, Reading, MA in 1963.

[5] K.B. Datta, Matrix and Linear Algebra, Prentice Hall of India, 1991.

[6] N. Gastinel, Linear Numerical Analysis, Academic Press, London, 1970.

[7] N.J. Higham, Estimating the matrix p-norm, Numer. Math. 62 (1992), 539-555.

[8] G.H. Golub and C.F. Van Loan, Matrix Computations, Second Edition, The Johns Hopkins University Press, Baltimore-London, 1989.

[9] W.S. Hall and M.L. Newell, The mean value theorem for vector valued functions: a simple proof, Math. Mag. 52 (1979), 157-158.

[10] R.E. Kalman, Algebraic Aspects of the Generalized Inverse of a Rectangular Matrix. In Generalized Inverses and Applications: Proceedings of an Advanced Seminar. Academic Press 1976, pp. 111-124.

[11] V. Rakočević, On continuity of the Moore-Penrose and Drazin Inverses, Mat.i Vesnik 39(3-4) (1997), 163-172.

[12] H. Schneider and W.G. Strang, Comparison theorems for supremum norms, Numer. Math. 4 (1962), 15-20.

[13] E. Saggini and M. Torrente, An Euclidean norm based criterion to assess robots' 2D pathfollowing performance, J. Algebr. Stat. 7(1) (2016), 45-71.

[14] J.R. Sendra and J. Sendra, Computation of Moore-Penrose Generalized Inverses of Matrices with Meromorphic Function Entries. Applied Mathematics and Computation 313C (2017) pp. 355-366.

[15] G.W. Stewart, On the continuity of the Generalized Inverse, SIAM J. Appl. Math., 1969, $\mathbf{1 7}(1), 33-45$.

[16] M. Torrente and M.C. Beltrametti, Almost vanishing polynomials and an application to the Hough transform, J. Algebra Appl. 13(8) (2014), 1450057 [39 pages].

[17] M. Torrente and M.C. Beltrametti, Corrigendum for the paper "Almost vanishing polynomials and an application to the Hough transform", arXiv:1705.00158v1 [math.AG].

[18] M. Torrente, M.C. Beltrametti, and J.R. Sendra, Perturbation of polynomials and applications to the Hough transform, J. Algebra, 486 (2017), 328-359.

[19] L.N. Trefethen and D. Bau, Numerical Linear Algebra, SIAM, 1997.

[20] H.F. Walker and L.T. Watson, Least-change secant update methods for underdetermined systems, SIAM J. Numer. Anal. 27(5) (1990), 1227-1262. 
M. Torrente, Istituto di Matematica Applicata e Tecnologie Informatiche "E. Magenes" CNR, Via de Marini, 6, I-16149 Genova, Italy. e-mail torrente@dima.unige.it

M.C. Beltrametti, Dipartimento di Matematica, Università di Genova, Via Dodecaneso 35, I-16146 Genova, Italy. e-mail beltrame@dima.unige.it

J.R. Sendra, Research Group ASYNACS. Dpto. de Física y Matemáticas, Universidad de Alcalá, 28871 Alcalá de Henares (Madrid), Spain. e-mail Rafael.sendra@uah.es 


\section{Memorandum of modifications of the revised version of " $r$-norm bounds and metric properties for zero loci of real analytic functions"}

We cordially thank the reviewers for their comments, and for the time devoted to analyze our work. We have followed all their suggestion and comments. 